\title{
Zenith angle distributions at Super-Kamiokande and SNO and the solution of the solar neutrino problem
}

\author{
M. C. Gonzalez-Garcia* and Carlos Peña-Garay ${ }^{\dagger}$ \\ Instituto de Física Corpuscular, Universitat de València - C.S.I.C., Edificio Institutos de Paterna, Apt 2085, 46071 València, Spain \\ Alexei Yu. Smirnov \\ International Center for Theoretical Physics, 34100 Trieste, Italy \\ and Institute for Nuclear Research, Russian Academy of Sciences, Moscow 117312, Russia
}

(Received 28 December 2000; published 20 April 2001)

\begin{abstract}
We have performed a detailed study of the zenith angle dependence of the regeneration factor and distributions of events at SNO and SK for different solutions of the solar neutrino problem. In particular, we discuss the oscillatory behavior and the synchronization effect in the distribution for the LMA solution, the parametric peak for the LOW solution, etc. A physical interpretation of the effects is given. We suggest a new binning of events which emphasizes the distinctive features of the zenith angle distributions for the different solutions. We also find the correlations between the integrated day-night asymmetry and the rates of events in different zenith angle bins. The study of these correlations strengthens the identification power of the analysis.
\end{abstract}

DOI: $10.1103 /$ PhysRevD.63.113004

PACS number(s): 12.15.Ff, 14.60.Pq, 26.65.+t, 96.60.Jw

\section{INTRODUCTION}

Oscillations of solar neutrinos in the matter of the Earth modify the neutrino signal detected during the night $[1-3]$. The integral characteristic of this effect is the day-night asymmetry:

$$
A_{\mathrm{DN}} \equiv 2 \frac{N-D}{N+D},
$$

where $N$ and $D$ are the night and the day event rates, averaged over the year, and corrected for the Earth orbit eccentricity.

In most of the cases, the Earth-matter effect leads to the regeneration of the $\nu_{e}$ flux, so the asymmetry is positive. Conversely, a negative asymmetry appears for the small mixing angle (SMA) solution at small mixing angles. There is a number of detailed studies of the asymmetry and its dependence on neutrino energy and oscillation parameters [1-14]. Asymmetries have also been studied for certain intervals of the zenith angles [6].

The observation of a day-night asymmetry will be the proof of the matter conversion solution of the solar neutrino problem, excluding the vacuum oscillation solutions. However, the measurement of the asymmetry alone may not allow to select among the three possible Mikheyev-SmirnovWolfenstein (MSW) solutions: Large mixing angle (LMA), SMA, and low mass, low probability (LOW). A negative asymmetry is evidence of the SMA solution. However the expected value of the effect is small $\left|A_{\mathrm{DN}}\right|<0.01$ and it will be very difficult to establish experimentally such a small deviation from zero. Furthermore, one can make certain conclusions confronting the value of the asymmetry with other solar neutrino data (rates, energy spectrum of the recoil elec-

\footnotetext{
*Electronic address: concha@flamenco.ific.uv.es

†Electronic address: penya@flamenco.ific.uv.es

‡Electronic address: smirnov@ictp.trieste.it
}

trons). In particular, a relatively large asymmetry will be inconsistent with other solar neutrino data (rates, recoil electron energy spectrum) in the case of SMA and LOW solutions, thus favoring the LMA solution.

Further insight can be obtained by studying the zenith angle distribution of events during the night. It turns out that different solutions lead to qualitatively different distributions. One of the first detailed studies of the zenith angle dependence of events for LMA, SMA, and LOW solutions of the solar neutrino problem was done by Baltz and Wenesser [7] and then further elaborated in $[4,8-11,14]$.

It was realized that for the SMA solution the distribution has a peculiar form with rather small effect for neutrinos crossing the mantle only $\left(\cos \theta_{Z}<0.837\right)$ and large regeneration effect (peak) for neutrinos whose trajectories crossed both the mantle and the core of the Earth $[7,9,10,15]$. This peak at large $\cos \theta_{Z}$ was interpreted as due to certain constructive interference of the oscillation effects in the mantle and in the core of the Earth [16] or in more simple and transparent way, as the effect of parametric enhancement of oscillations [17] (see also [18,19], and for later discussion $[20,21])$.

For the LMA solution one expects averaging of oscillations due to integration over the energies of neutrino and detected charge lepton as well as over finite size of the zenith angle bins. This leads to a rather flat distribution with small variations of the average rate during the night $[7,11-13]$. A significant regeneration effect is expected already in the first night bin $\cos \theta_{Z}<0.2$.

For the LOW solution one gets the highest rate in the second night bin $[7,14]$, followed by a dip and then again an increase of the rate for large $\cos \theta_{Z}$. This peak has a simple interpretation as the oscillation maximum which corresponds to the phase of oscillations $\phi=\pi$.

Thus, a detailed study of the zenith angle distribution will allow to disentangle the solutions. Moreover, it may allow to determine the oscillation parameters. Measurements of the zenith angle distribution are also interesting because they 
open the possibility to study various matter oscillation effects, such as oscillations in matter with constant and slowly changing density, adiabatic conversion, effects of the resonance enhancement of oscillations, parametric effects, oscillation effects in thin layers (with small density width), and effects of small density jumps, etc.

Furthermore, in principle, (if the oscillation parameters are determined from some other experiments) the precise measurement of the zenith angle distribution will allow to check models of the Earth density profile.

In this paper we continue to study in detail the zenith angle distributions for the different MSW solutions of the solar neutrino problem. We give physical interpretation of various features of the distributions. We clarify what can be learned besides identification of the solution of the solar neutrino problem by measuring the zenith angle distributions in the present and in future high statistics experiments.

The paper is organized as follows: in Sec. II we present general expressions for the survival probability and the regeneration factor which describe the Earth-matter effect and we study the properties of the regeneration factor in the constant and slowly changing density approximation. In Sec. III we present the results of our numerical calculations of the regeneration factor and the rates for the Super-Kamiokande and SNO for a realistic Earth-matter profile. We give an interpretation of the results of these calculations using the results of the analytical studies. In Sec. IV we propose a new $\cos \theta_{Z}$ binning which emphasizes distinctive features of the distributions for the different solutions. We also study the correlations of rates as well as ratios of rates with the value of the day-night asymmetry as a way to strengthen the identification power of analysis. We summarize and discuss our results in Sec. V.

\section{GENERAL RELATIONS}

\section{A. Regeneration factor and conversion inside the Sun}

For the range of oscillation parameters of interest $\left(\Delta m^{2}\right.$ $>10^{-8} \mathrm{eV}^{2}$ ), the probability $P_{e e}$ to detect the solar electron neutrino at a detector can be written as follows:

$$
P_{e e}=P_{1}+\left(1-2 P_{1}\right)\left(\sin ^{2} \theta+f_{\text {reg }}\right) .
$$

In Eq. (2) the Earth regeneration factor, $f_{\text {reg }}$, is defined as [14]

$$
f_{\text {reg }} \equiv P_{2 e}-\sin ^{2} \theta,
$$

where $P_{2 e}$ is the probability of the $\nu_{2} \rightarrow \nu_{e}$ conversion inside the Earth. In the absence of the Earth-matter effect we have $P_{2 e}=\sin ^{2} \theta$, so that $f_{\text {reg }}=0$.

In Eq. (2) $P_{1}$ is the probability of the $\nu_{e} \rightarrow \nu_{1}$ conversion inside the Sun which can be approximated by the well known expression

$$
P_{1}=\frac{1}{2}+\left(\frac{1}{2}-P_{c}\right) \cos 2 \theta_{S} .
$$

Here $\theta_{S}$ is the matter mixing angle at the production point inside the Sun:

$$
\cos 2 \theta_{S} \equiv \cos 2 \theta_{m}\left(\rho_{S}\right),
$$

where $\theta_{m}(\rho)$ is the mixing angle in matter determined by

$$
\cos 2 \theta_{m}=\frac{-1+\eta \cos 2 \theta}{\left(1-2 \eta \cos 2 \theta+\eta^{2}\right)^{1 / 2}} .
$$

Here

$$
\eta \equiv \frac{l_{0}}{l_{\nu}}=\frac{\sqrt{2} m_{N}}{G_{F} \rho Y_{e}} \frac{\Delta m^{2}}{E}=0.66\left(\frac{\Delta m^{2} / E}{10^{-13} \mathrm{eV}}\right)\left(\frac{1 \mathrm{~g} \mathrm{~cm}^{-3}}{\rho Y_{e}}\right)
$$

is the ratio between the refraction length, $l_{0}$, and the neutrino oscillation length in vacuum, $l_{\nu}$ :

$$
l_{0} \equiv \frac{2 \pi m_{N}}{\sqrt{2} G_{F} \rho Y_{e}}, \quad l_{\nu} \equiv \frac{4 \pi E}{\Delta m^{2}} .
$$

In Eqs. (7) and (8) $\rho$ is the matter density, $Y_{e}$ is the number of electrons per nucleon and $m_{N}$ is the nucleon mass. $P_{c}$ is the jump probability which takes for an exponential density profile the following form $[22,23]$ :

$$
P_{c}=\frac{e^{-\gamma \sin ^{2} \theta}-e^{-\gamma}}{1-e^{-\gamma}},
$$

where $\gamma$ is the ratio of the density scale height $l_{\rho}$ and the neutrino oscillation length:

$$
\gamma \equiv \frac{4 \pi^{2} l_{\rho}}{l_{\nu}}=1.05\left(\frac{\Delta m^{2} / E}{10^{-15} \mathrm{eV}}\right)\left(\frac{l_{\rho}}{r_{0}}\right), \quad l_{\rho} \equiv \frac{\rho}{d \rho / d r} .
$$

$r_{0}=R_{\odot} / 10.54$ is the height scale in the exponential approximation to the solar density profile. Inserting Eq. (4) into Eq. (2) we get

$$
P_{e e}=P_{D}-\left(1-2 P_{c}\right) \cos 2 \theta_{S} f_{\mathrm{reg}},
$$

where

$$
P_{D}=\frac{1}{2}+\frac{1}{2}\left(1-2 P_{c}\right) \cos 2 \theta_{S} \cos 2 \theta
$$

is the survival probability in the absence of the Earth-matter effect, i.e., during the day.

According to Eq. (11), the regeneration effect appears multiplied by two factors.

(1) The adiabaticity factor $\left(1-2 P_{c}\right)$, which describes the adiabaticity of the conversion inside the Sun. The factor is maximal for $P_{c}=0$, that is, for the case of pure adiabatic propagation and it decreases with the increase of the adiabaticity breaking. For $P_{c}=1 / 2$ the factor and the regeneration effect are zero, and at $P_{c}<1 / 2$ the Earth-matter effect changes the sign.

(2) The resonance position factor $\cos 2 \theta_{S}$, which determines how far the resonance layer is situated from the production point (in the density scale). When neutrinos are pro- 
duced at the resonance, one has $\cos 2 \theta_{S}=0$, while $\cos 2 \theta_{S}$ $<0$ if the resonance occurs at densities lower than the one at the production point. The parameter $\cos 2 \theta_{S}$ decreases with the resonance density, and it reaches the value $\cos 2 \theta_{S} \approx-1$ when the resonance layer is sufficiently far out from the production region (i.e., at much lower densities).

From the previous discussion we conclude that the largest regeneration effect occurs when the neutrino propagation is adiabatic and the resonance happens far from the production region.

From Eq. (11) one can obtain the daily average survival probability which for $\eta \ll 1\left(\cos 2 \theta_{S} \approx-1\right)$ takes the form

$$
\bar{P} \equiv \frac{1}{2}\left(P_{D}+P_{N}\right)=\frac{1}{2}\left[1+\left(1-2 P_{1}\right)\left(f_{\text {reg }}-\cos 2 \theta\right)\right] .
$$

The numbers of events during the day and during the night are proportional to averaged probabilities $P_{D}$ and $P_{N}$, therefore the day-night asymmetry (1) can be written as

$$
A_{\mathrm{DN}} \equiv \frac{P_{N}-P_{D}}{\bar{P}}=\frac{2 f_{\mathrm{reg}}}{1 /\left(1-2 P_{1}\right)-\cos 2 \theta+f_{\mathrm{reg}}},
$$

where both in Eq. (13) and Eq. (14), $f_{\text {reg }}$ should be averaged over the neutrino trajectories during the night.

\section{B. Regeneration factor in constant density and adiabatic approximations}

To obtain the zenith angle distributions we have performed exact numerical calculations of the regeneration factor integrating the evolution equation in the Earth-matter with the Earth density profile given in the Preliminary Reference Earth Model (PREM) [25]. However, a number of qualitative features of our results can be easily understood in the simplified two-layers approximation of the Earth profile. In this approximation the profile consists of the mantle and the core with slowly changing densities and a sharp change of the density between the layers. In what follows we will parametrize the zenith angle dependence in terms of $\left|\cos \theta_{Z}\right|$ which, for simplicity, we will write omitting the moduli. Equivalently, this would correspond to $\theta_{Z}$ being the nadir angle. We call the region of zenith angles $\cos \theta_{Z}=0-0.837$, for which neutrinos cross the mantle only, the mantle region and the region $\cos \theta_{Z}=0.837-1$, for which neutrinos cross both the mantle and the core, the core region.

Simple analytical results can be obtained in the constant density approximation, or, in general, in the adiabatic approximation. In the case of constant density (which would correspond to neutrinos crossing the mantle at small enough $\cos \theta_{Z}$ ) we obtain the following expression for the regeneration factor:

$$
f_{\text {reg }}=\sin 2 \theta_{m} \sin \left(2 \theta_{m}-2 \theta\right) \sin ^{2}\left(\frac{\pi d}{l_{m}}\right),
$$

where $\theta_{m}$ is the mixing angle in the Earth matter given in Eq. (6) with $\eta$ evaluated for the Earth density, and $d$ is the width of the layer or equivalently the distance travelled by the neutrino. Using the expression for the mixing angle (6) we get that

$$
f_{\text {reg }}=\frac{\eta \sin ^{2} 2 \theta}{\left(1-2 \eta \cos 2 \theta+\eta^{2}\right)} \sin ^{2}\left(\frac{\pi d}{l_{m}}\right)
$$

which can be rewritten in the following form (see also [7]):

$$
f_{\text {reg }}=\frac{1}{\eta} \sin ^{2} 2 \theta_{m} \sin ^{2}\left(\frac{\pi d}{l_{m}}\right) .
$$

The oscillation length in matter, $l_{m}$, equals

$$
\begin{aligned}
l_{m} & =l_{\nu} \frac{\sin 2 \theta_{m}}{\sin 2 \theta}=l_{\nu} \frac{\eta}{\left(1-2 \eta \cos 2 \theta+\eta^{2}\right)^{1 / 2}} \\
& =\frac{l_{0}}{\left(1-2 \eta \cos 2 \theta+\eta^{2}\right)^{1 / 2}},
\end{aligned}
$$

where $l_{0}$ and $l_{\nu}$ are defined in Eq. (8) and $\eta$ should be taken for the Earth density.

Written in the form of Eq. (17), $f_{\text {reg }}$ differs from the expression for the probability of usual flavor oscillations in matter by the factor $1 / \eta \propto n_{e} E / \Delta m^{2}$. One can notice as well that the amplitude of oscillations of $f_{\text {reg }}$

$$
A_{f}=\frac{1}{\eta} \sin ^{2} 2 \theta_{m}
$$

is symmetric with respect to the exchange $\eta \leftrightarrow 1 / \eta$, as can be seen explicitly from the Eq. (16). Due to the additional factor $1 / \eta$ the asymptotics of the oscillation amplitude are $A_{f} \rightarrow 0$ both at $\eta \rightarrow 0$ and at $\eta \rightarrow \infty$ which differ from the ones for flavor oscillations [1].

According to Eq. (17), in the case of one layer with constant density, the regeneration $f_{\text {reg }}$ is always (for any value $\Delta m^{2} / E$ and $\theta$ ) positive, i.e., the matter effect of Earth always enhances the survival probability $P_{e e}$ (see Appendix).

In fact, in the mantle (and also in the core) the density changes rather significantly. According to the preliminary reference Earth model (PREM) it increases from $\rho \sim 3.2$ $\mathrm{g} / \mathrm{cm}^{3}$ near the surface of the Earth to $\rho \sim 5.6 \mathrm{~g} / \mathrm{cm}^{3}$ at the border with the core. The density changes smoothly apart from several jumps at distances $(0.05-0.12) R_{E}$ from the surface. Such a density variation leads to deviation from the simple oscillation picture described above. One expects certain interplay of the oscillations and the adiabatic evolution which results in the change of the oscillation probability from the constant density description. Furthermore the small jumps in the density may induce some irregularities in the behavior of the zenith angle distribution.

The description of the effects is simple if the adiabaticity condition is fulfilled (as it happens for the LMA solution). In this case the average probability and the amplitude of oscillations are determined uniquely by the instantaneous value of the density. Therefore $\bar{P}$ and the depth of oscillations of $f_{\text {reg }}$ will be determined by the density at the surface $\rho_{s}$ : 


$$
f_{\text {reg }}=\frac{1}{\eta\left(\rho_{s}\right)} \sin ^{2} 2 \theta_{m}\left(\rho_{s}\right) \sin ^{2}\left(\pi \int \frac{d x}{l_{m}}\right)
$$

In some cases when adiabaticity is violated one can describe the results using constant density approximation with some effective value of the density for each trajectory:

$$
l_{m}=l_{m}\left(\theta_{Z}\right), \quad \theta_{m}=\theta_{m}\left(\theta_{Z}\right), \quad \eta=\eta\left(\theta_{Z}\right) .
$$

If the oscillations are averaged due to integration over the energy or/and the zenith angle we get

$$
\bar{f}_{\text {reg }}=\frac{\eta \sin ^{2} 2 \theta}{2\left(1-2 \eta \cos 2 \theta+\eta^{2}\right)}
$$

which coincides with expression used in [14] with $\eta$ taking an average value for the corresponding $E$ and $\theta_{Z}$ range.

In the case of the core crossing trajectories the description becomes more complicated [30,16,17,20,21].

The analytical results presented in this section allow us to get a straightforward interpretation of the results of the numerical computations. Moreover, they give the correct functional dependence of observables on the neutrino parameters.

\section{ZENITH ANGLE DEPENDENCE OF THE REGENERATION FACTORS AND RATES}

In this section we consider the zenith angle dependence of the survival probability in Eq. (2), the regeneration factor in Eq. (3), and the event rates at SuperKamiokande (SK) and SNO for oscillation parameters from the LMA, LOW, and SMA solutions to the solar neutrino problem.

The reduced rate of the electron scattering (ES) events at Super-Kamiokande is defined as

$$
\begin{aligned}
{[\mathrm{ES}] \equiv } & \frac{N_{E S}}{N_{E S}^{S S M}} \\
= & \frac{1}{N_{\nu e}} \int_{E_{t h}} d E_{e}^{\prime} R\left(E_{e}, E_{e}^{\prime}\right) \int d E_{\nu} F\left(E_{\nu}\right) \sigma_{e}\left(E_{\nu}, E_{e}^{\prime}\right) \\
& \times\left[P_{e e}\left(E_{\nu}, \theta_{Z}\right)+r\left(E_{\nu}, E_{e}^{\prime}\right)\left(1-P_{e e}\left(E_{\nu}, \theta_{Z}\right)\right),\right.
\end{aligned}
$$

where $R\left(E_{e}, E_{e}^{\prime}\right)$ is the energy resolution factor, $F\left(E_{\nu}\right)$ is the flux of the electron neutrinos without oscillations, $\sigma_{e}$ is the $\nu_{e}-e$ elastic scattering cross section and $r\left(E_{\nu}, E_{e}^{\prime}\right)$ $\equiv \sigma_{\mu} / \sigma_{e}$. with $\sigma_{\mu}$ being the $\nu_{\mu}-e$ elastic scattering cross section [26]. $N_{\nu e}$ is the normalization factor which equals the integral in Eq. (23) taken at $P=1$.

The reduced rate of the charged current (CC) events at SNO is obtained from

$$
\begin{aligned}
{[\mathrm{CC}] \equiv \frac{N_{C C}}{N_{C C}^{S S M}}=} & \frac{1}{N_{\nu d}} \int_{E_{t h}} d E_{e}^{\prime} R\left(E_{e}, E_{e}^{\prime}\right) \int d E_{\nu} F\left(E_{\nu}\right) \\
& \times \sigma_{C C}\left(E_{\nu}, E_{e}^{\prime}\right) P_{e e}\left(E_{\nu}, \theta_{Z}\right)
\end{aligned}
$$

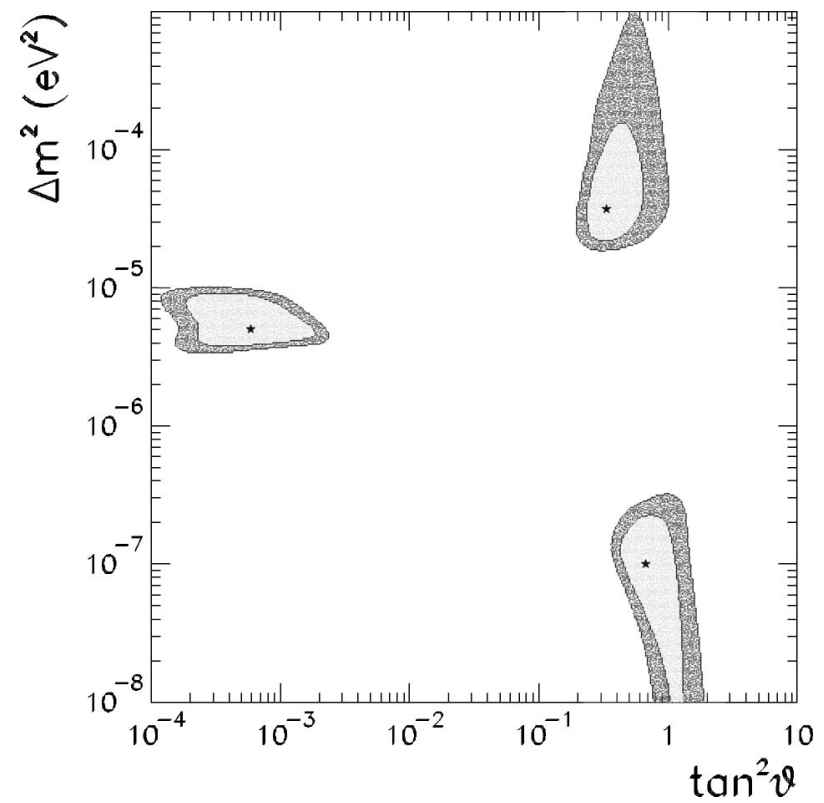

FIG. 1. Regions of solutions of the solar neutrino problem in the $\Delta m^{2}-\tan ^{2} \theta$ plane. Dots correspond to the best fit points for each type of the solution. Contours show 90\% C.L. regions (light) and 99\% C.L. regions (dark) found with respect to local minima for each region.

where $\sigma_{C C}$ is the CC neutrino-deuteron cross section [27], and $N_{\nu d}$ is the normalization factor which equals the integral in Eq. (24) taken at $P=1$.

The results presented in this section have been obtained for oscillation parameters in the presently allowed regions of solutions of the solar neutrino problem. In Fig. 1 we show the results of the global fit to the data which include (i) the SuperKamiokande (SK) data after 1117 days of operation (total number of events and day and night energy spectra), (ii) Gallex, GNO, and SAGE data, (iii) the Homestake data. Shown are the best fit points for each solution as well as $90 \%$ and $99 \%$ C.L. regions found from the local minima in each allowed region. We show the solutions only in the range $\Delta m^{2}>10^{-8} \mathrm{eV}^{2}$ for which Earth-matter effects on the boron neutrinos can be substantial. The fit includes the latest standard solar model fluxes, the 2000 Bahcall-Basu-Pinsonneault (BBPOO) model [28]. For details of the statistical analysis applied to the different observables we refer to Ref. [29].

The results for the event rates presented in Figs. 3-10 have been normalized to the SK total rate. That is, for each set of the oscillation parameters, expressions in Eqs. (23) and (24) have been multiplied by a boron flux normalization factor $f_{S K}$ in order get value of [ES] as it is measured at SK.

\section{A. LMA}

In the LMA region, the adiabaticity condition is satisfied and we can safely put $P_{c}=0$. Consequently Eq. (11) simplifies to

$$
P_{e e}=P_{D}-\cos 2 \theta_{S} f_{\text {reg }}
$$



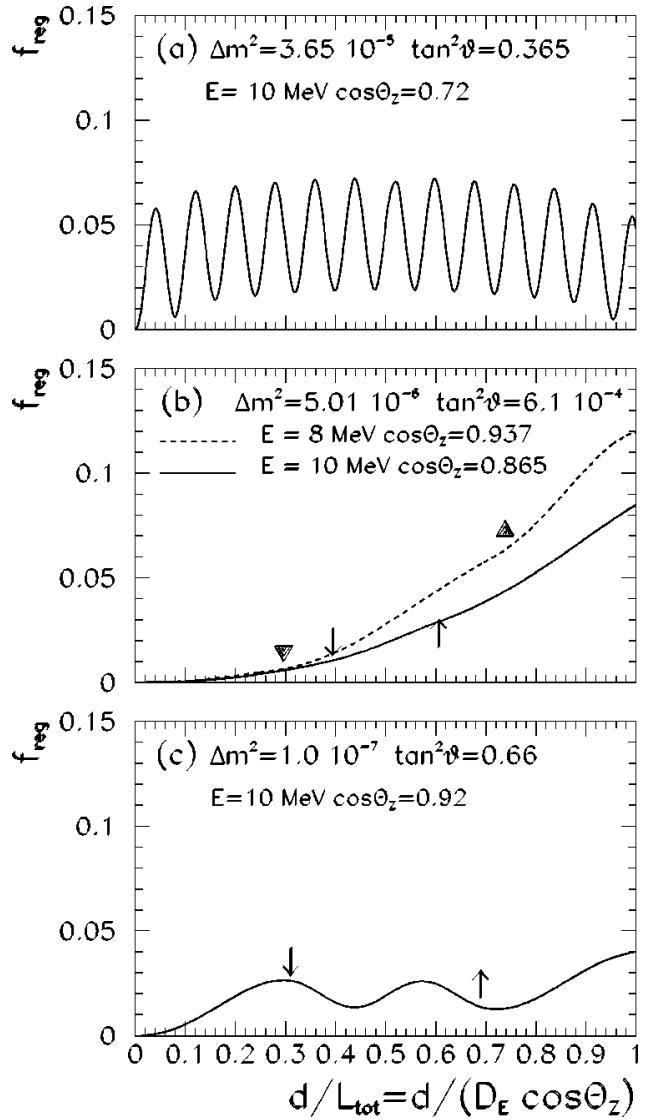

FIG. 2. The dependence of the regeneration factor on distance in units of the total length of a trajectory for a given value of $\cos \theta_{Z}$ and for the oscillation parameters indicated in the panels. The dependence is shown for (a) LMA solution, (b) SMA solution for two different energies (arrow and triangles indicate the point when neutrino enters and leave the core), and (c) LOW solution.

$$
P_{D}=\frac{1}{2}-\frac{1}{2} \cos 2 \theta_{S} \cos 2 \theta
$$

Moreover, in the low $\Delta m^{2}$ part of the LMA region we can take $\cos 2 \theta_{S} \approx-1$, so that

$$
P_{e e}=\sin ^{2} \theta+f_{\text {reg }} \quad\left(\Delta m^{2} \ll 10^{-4} \mathrm{eV}^{2}\right) .
$$

Let us discuss first the behavior of the regeneration factor. In Figs. 3(a) and 4(a) we show the zenith angle dependence of the $f_{\text {reg }}$ for different values of neutrino energies. In the interval $\cos \theta_{Z}=0-0.837$ the dependence has a quasiperiodical shape. The amplitude of oscillation slightly changes with $\cos \theta_{Z}$ as a consequence of the adiabaticity violation (arising from the small jumps of the density). At $\cos \theta_{Z}>0.837$ the dependence is more irregular due to the Earth core effect. All these features, as well as the dependence of the regeneration factor on the neutrino parameters (energy, mixing angle, and $\Delta m^{2}$ ) can be immediately understood from our analytical consideration as we discuss next.

In the LMA region of oscillation parameters the Earthmatter effects can be considered as neutrino oscillations in matter with slowly changing density. Moreover, in this region $\eta>3$ [for the present best fit point (see Fig. 3) and $E$

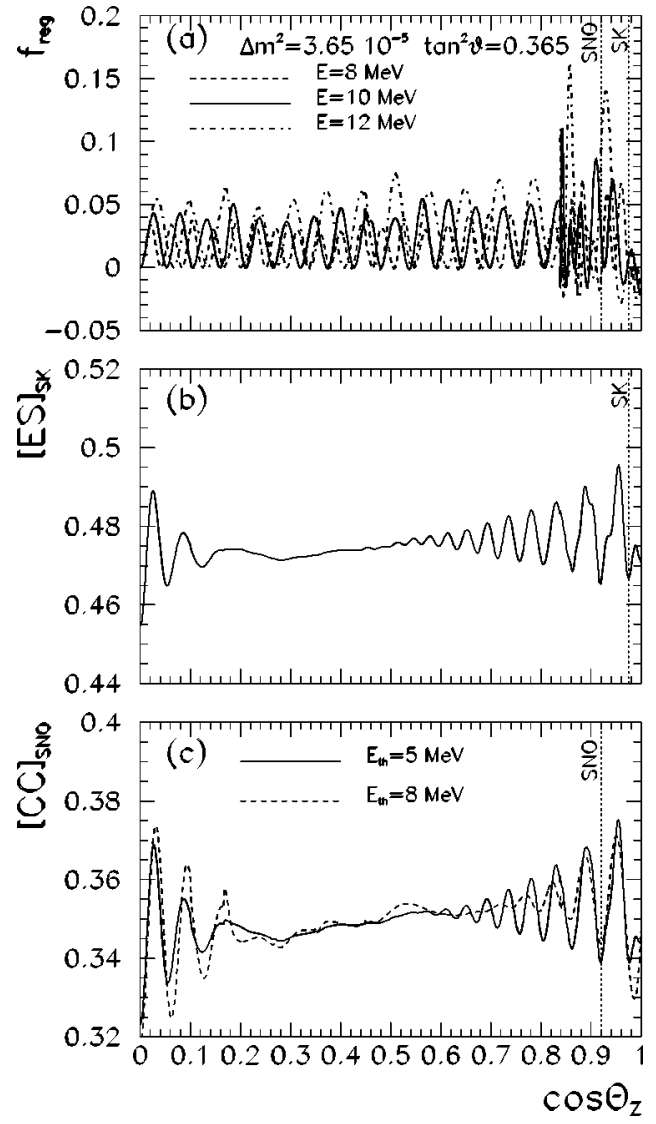

FIG. 3. The zenith angle distributions for the best fit point $\left(\Delta m^{2}=3.25 \times 10^{-5} \mathrm{eV}^{2}, \tan ^{2} \theta=0.365\right)$ in the LMA solution. (a) The dependence of the regeneration factor on $\cos \theta_{Z}$ for different neutrino energies as labeled in the figure. Vertical dashed lines indicate the maximal values of $\cos \theta_{Z}$ which can be realized at SK and SNO. (b) The zenith angle dependence of the $\nu e$ event rate at Super-Kamiokande above the energy $E_{t h}=5.5 \mathrm{MeV}$. (c) The zenith angle dependence of the $\nu d$ charged current event rate at SNO for two different energy thresholds (5 and $8 \mathrm{MeV}$ ).

$\sim 10 \mathrm{MeV}$ we get $\eta=12]$. This means that oscillations in the Earth with parameters from the LMA region proceed in the vacuum dominated regime, when the matter effect gives relatively small corrections. In particular, the oscillation length is close to the vacuum oscillation length:

$$
l_{m} \approx l_{\nu}\left[1+\frac{2 \cos 2 \theta}{\eta}\right]
$$

which holds to first order in $1 / \eta$ and presents a rather weak dependence on the mixing angle. For the best fit point oscillation parameters and $\rho Y_{e} \sim 2 \mathrm{~g} / \mathrm{cc}$ we get

$$
l_{m} \approx 10^{3} \mathrm{~km}\left(\frac{E}{10 \mathrm{MeV}}\right) \text {. }
$$

So, the oscillation length is much shorter than the diameter of the Earth for all the relevant oscillation parameters.

The expression for the mixing angle can be approximated as 

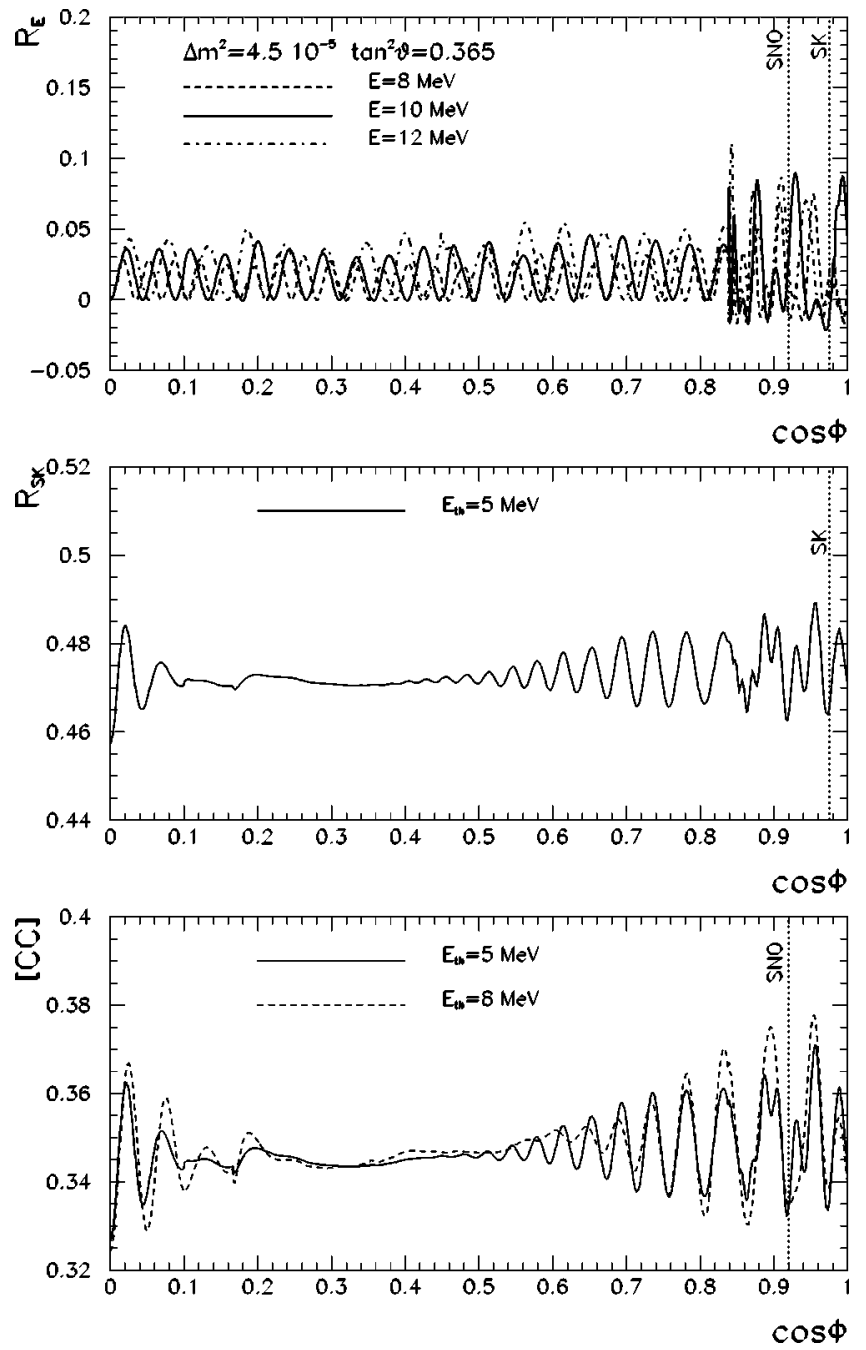
$\left.\mathrm{eV}^{2}\right)$

FIG. 4. Same as Fig. 3 for a larger $\Delta m^{2}\left(\Delta m^{2}=4.5 \times 10^{-5}\right.$

$$
\tan 2 \theta_{m} \approx \tan 2 \theta\left[1+\frac{1}{\cos 2 \theta \eta}\right]
$$

and the oscillation phase acquired by neutrinos crossing the Earth equals

$$
\phi \equiv \frac{2 \pi d\left(\theta_{Z}\right)}{l_{m}\left(\theta_{Z}\right)} \approx \frac{2 \pi D \cos \theta_{Z}}{l_{\nu}},
$$

where $D=1.3 \times 10^{4} \mathrm{~km}$ is the diameter of the Earth. That is, the phase increases linearly with $\cos \theta_{Z}$, and therefore, $f_{\text {reg }}$ turns out to be an almost periodical function of $\cos \theta_{Z}$. Also, the phase is inversely proportional to the energy, so that the number of periods increases as $1 / E$. The period of oscillations in the $\cos \theta_{Z}$ scale equals $T\left(\cos \theta_{Z}\right) \approx l_{\nu} / D$.

In Fig. 2 we show the dependence of $f_{\text {reg }}$ on the distance travelled by the neutrino inside the Earth for some trajectories and for best fit values of the oscillation parameters. For LMA the oscillation length is much smaller than the typical scale of the density change:
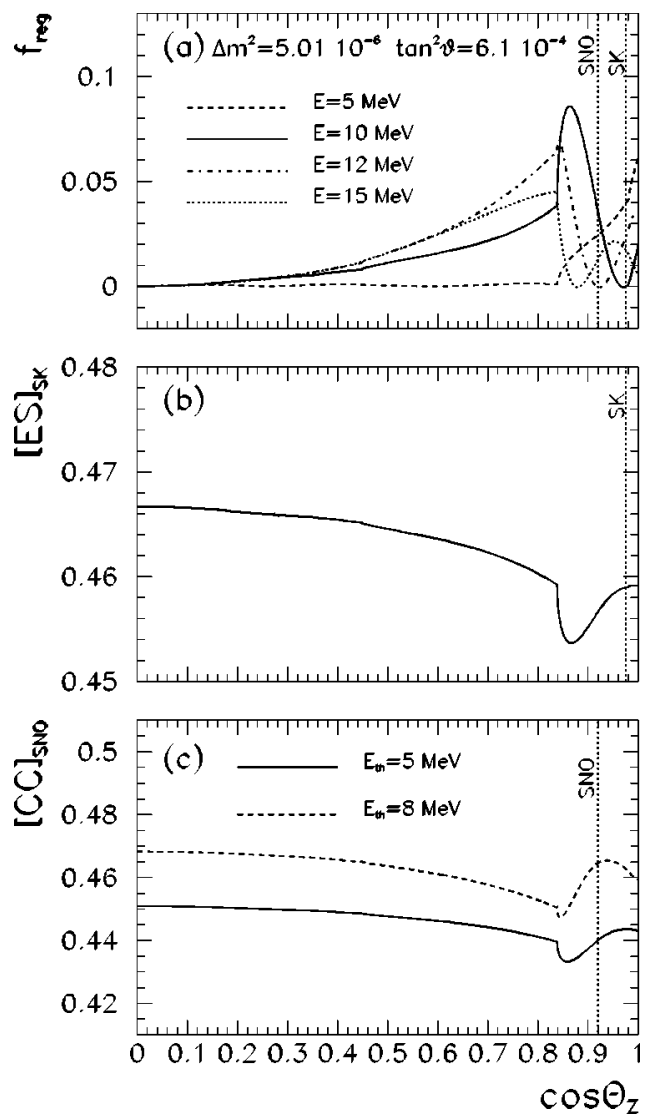

FIG. 5. Same as Fig. 3 for the best fit point $\left(\Delta m^{2}\right.$ $\left.=5.01 \times 10^{-6} \mathrm{eV}^{2}, \tan ^{2} \theta=6.1 \times 10^{-4}\right)$ in the SMA solution.

$$
l_{\rho} \equiv \rho\left(\frac{d \rho}{d x}\right)^{-1} \sim \frac{D}{2}
$$

both for the mantle and for the core: $l_{m} \ll l_{\rho}$. Since the mixing angle is large this leads to good adiabaticity (especially far from the resonance). Small density jumps can be treated then as small perturbations. As a result, the average $f_{\text {reg }}$, $\overline{f_{\text {reg }}}$, follows the density change. Both $\overline{f_{\text {reg }}}$ and the amplitude of oscillations increase towards the center of the trajectory where the density is maximal. Since the profile is symmetric (with respect to the middle point of the trajectory), the dependence of $\overline{f_{\text {reg }}}$ and $A_{f}$ on distance is also symmetric. At the detector $\overline{f_{\text {reg }}}$ and $A_{f}$ are determined by the surface density $(\rho \sim 3.2 \mathrm{~g} / \mathrm{cc})$. Therefore in the adiabatic approximation the amplitude of the oscillatory behavior of $f_{\text {reg }}$ should not depend on $\cos \theta_{Z}$. The variations of the amplitude $A_{f}$ with $\cos \theta_{Z}$ seen in Fig. 2 are produced by the small density jumps which violate the adiabaticity.

As follows from Eq. (19), the amplitude of oscillations takes the form

$$
A_{f} \approx \frac{\sin ^{2} 2 \theta}{\eta_{s}} k_{n a}\left(\theta_{Z}\right) \approx \frac{E \rho_{s}}{\Delta m^{2}},
$$

where $\eta_{s}$ and $\rho_{s}$ are the value of parameter $\eta$ and the density at the surface of the Earth and $k_{n a} \sim 1$ is the parameter which 

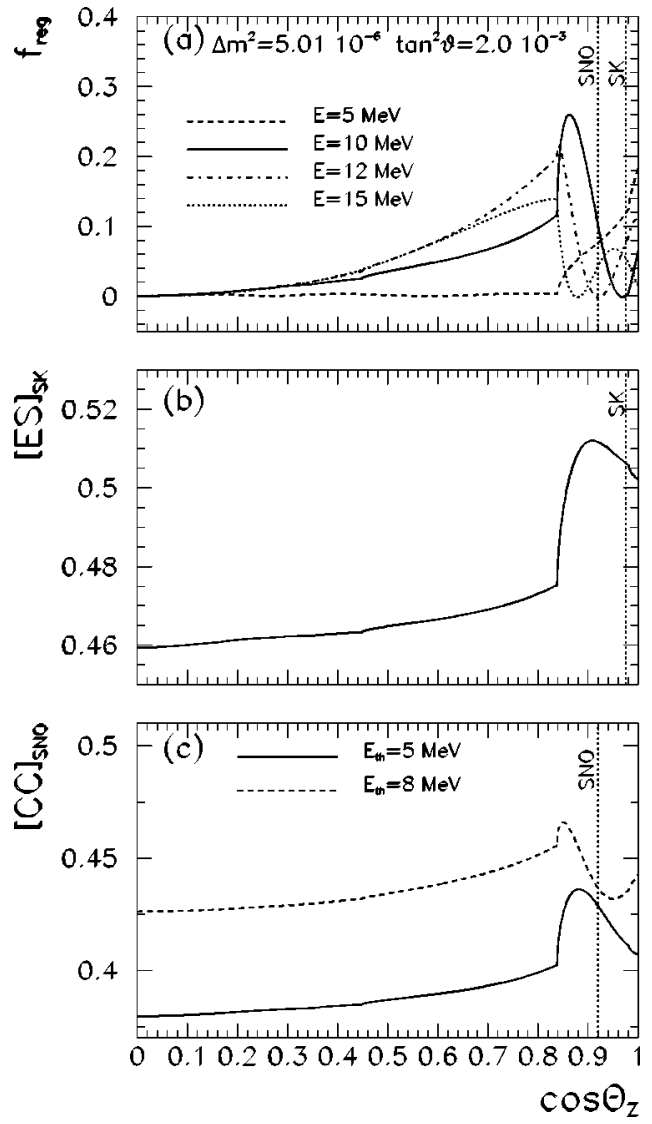

FIG. 6. Same as Fig. 5 for a larger mixing $\left(\tan ^{2} \theta\right.$ $=2.0 \times 10^{-3}$ ) in the SMA solution.

describes small effects of the adiabaticity violation. Thus, the amplitude increases with energy, as can be also seen from Figs. 3(a) and 4(a), and it is inversely proportional to $\Delta m^{2}$. The amplitude is proportional to $\sin ^{2} 2 \theta$, however variations of this parameter in the LMA region produces small changes specially in the near maximal mixing region.

Combining the results of Eqs. (31) and (33) we get the zenith angle dependence of the regeneration factor in the mantle region

$$
f_{\text {reg }} \approx \frac{\sin ^{2} 2 \theta}{\eta_{s}} k_{n a}\left(\theta_{Z}\right) \sin ^{2} \frac{\pi D \cos \theta_{Z}}{l_{\nu}} .
$$

The zenith angle dependence becomes more complicated in the region $\cos \theta_{Z}>0.837$, when neutrino trajectories cross the core of the Earth $[30,16,17,20,21]$. This is related to the appearance of the parametric (enhancement and suppression) effects on the top of the resonance enhancement in the core. Notice that for core-crossing trajectories the regeneration factor can be negative. In contrast, in the mantle region it is always positive as can be seen from Eq. (34) (see also Appendix).

Finally let us point out that the results shown in Figs. 3-7 for the regeneration factor are independent of the experimental setup. However the range of zenith angles covered by a given detector depends on its latitude: for SK $\cos \theta_{Z}^{\max }$
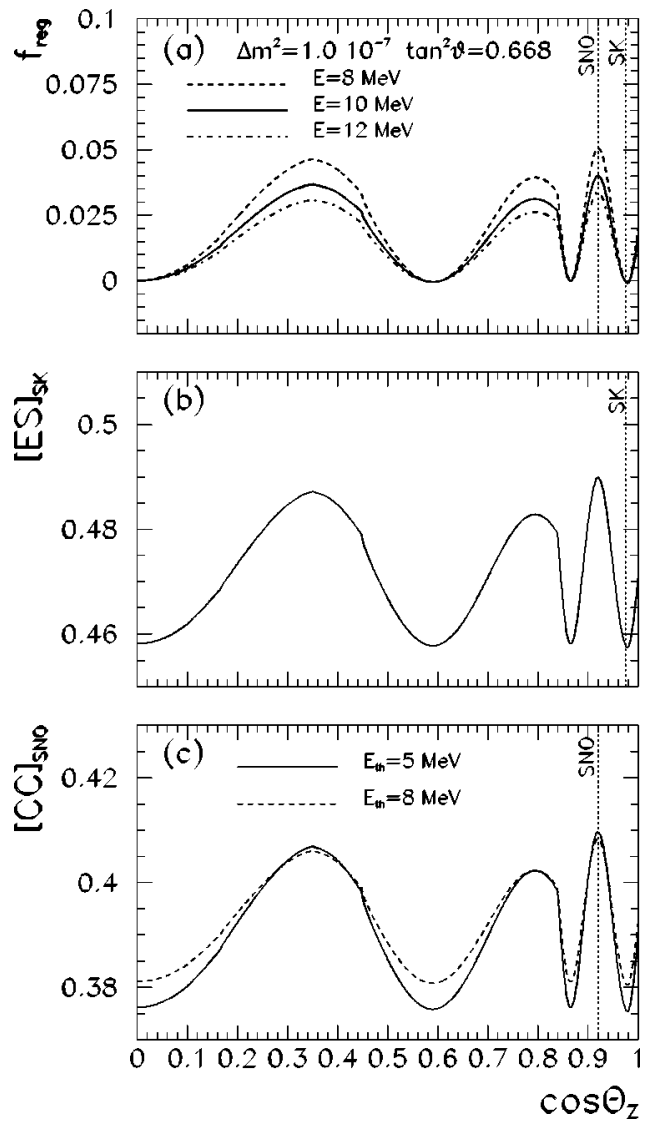

FIG. 7. Same as Fig. 3 for the best fit $\left(\Delta m^{2}=1.0 \times 10^{-7} \mathrm{eV}^{2}\right.$, $\left.\tan ^{2} \theta=0.668\right)$ in the LOW solution.

$=0.975$, whereas for the SNO $\cos \theta_{Z}^{\max }=0.92$. For the sake of clarity in Figs. 3-7 we mark those limiting zenith angles.

Let us now consider the zenith angle dependence of the event rates. We have calculated the rates above a given energy threshold using Eqs. (23) and (24). The calculation of rates involves folding of the survival probability with neutrino cross section, the flux of neutrinos and integration over the energy above threshold. Therefore results are different for SK and SNO.

In the lower part of the LMA region the survival probability is simply given by the sum of $\sin ^{2} \theta$ and the regeneration factor as seen in Eq. (27). Consequently, the [CC] rate can be written as

$$
[\mathrm{CC}] \approx \sin ^{2} \theta+\left\langle f_{\mathrm{reg}}\left(\theta_{Z}\right)\right\rangle_{C C}
$$

where the averaged regeneration factor is given by Eq. (24) with $P_{e e}$ substituted by $f_{\text {reg }}$.

Similarly, the [ES] rate can be expressed as

$$
[\mathrm{ES}] \approx \sin ^{2} \theta+r \cos ^{2} \theta+(1-r)\left\langle f_{\mathrm{reg}}\left(\theta_{Z}\right)\right\rangle_{E S},
$$

where $\left\langle f_{\text {reg }}\left(\theta_{Z}\right)\right\rangle_{E S}$ is given by Eq. (23) with $P_{e e}$ substituted by $f_{\text {reg }}$. Notice that the interactions of $\nu_{\mu}$ and $\nu_{\tau}$ enhance the average [ES] rate in comparison with [CC] rate giving an additional term $r \cos ^{2} \theta$, and they suppress the regeneration term by a factor $(1-r)$. 

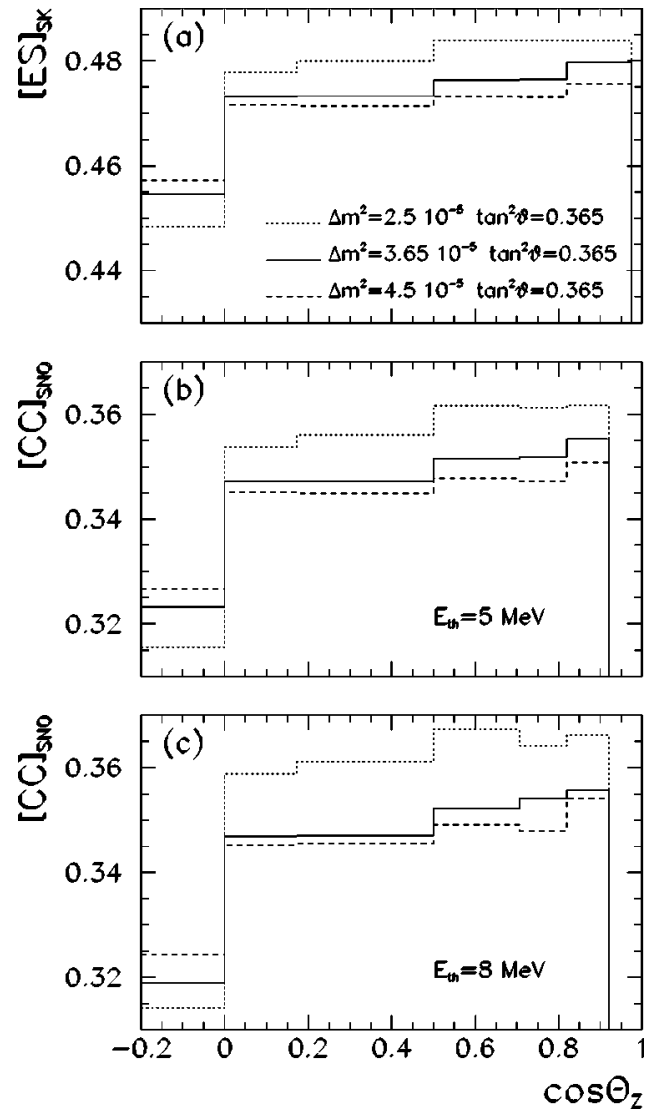

FIG. 8. The binned zenith angle dependence of (a) [ES] event rates at $\mathrm{SK}$ and (b) the $\nu d \mathrm{CC}$-event rate at $\mathrm{SNO}$ above at $E_{t h}=5$ $\mathrm{MeV}$ and (c) $E_{t h}=8 \mathrm{MeV}$ in the LMA region. The histograms correspond to different values of the oscillation parameters as labeled in the figure. The first bin corresponds to the day rate. The event rates have been computed with a boron flux normalization chosen to fit the measured rate at SK.

In Figs. 3(b) and 4(b) we plot the rate [ES] at SK as a function of $\cos \theta_{Z}$. The dependence of the reduced [CC] rate at $\mathrm{SNO}$ on $\cos \theta_{Z}$ for two different thresholds is shown in Figs. 3(c) and 4(c). (Similar figures have been obtained in $[7,4]$.) As seen in the figures, the rates are oscillating functions of $\cos \theta_{Z}$, however in contrast with the case of the regeneration factors, the amplitude of these oscillations changes with the zenith angle significantly. The amplitude is maximal at small $\cos \theta_{Z}\left(\cos \theta_{Z}=0-0.2\right)$, it decreases with $\cos \theta_{Z}$ till $\cos \theta_{Z} \sim 0.5$, and then it increases again. The first maximum is achieved already at $\cos \theta_{Z}=0.02-0.03$ which corresponds to the distances $250-400 \mathrm{~km}$. This large matter effect on small distances is related to the fact that the initial state is not a flavor state but an incoherent admixture of the mass eigenstates $\nu_{1}$ and $\nu_{2}$ [24]. Also the period of oscillations changes: it is about 0.07 for small $\cos \theta_{Z}$ and it decreases down to 0.05 for $\cos \theta_{Z}>0.6$. This important feature is related to the integration over the neutrino energy and it can be explained as the synchronization effect of the "waves" (oscillatory curves) $f_{\text {reg }}\left(\theta_{Z}\right)$ corresponding to different neutrino energies as we discuss next.

Let us notice that the boron neutrino flux is maximal at $E=8 \mathrm{MeV}$ and it decreases with energy, while both the cross
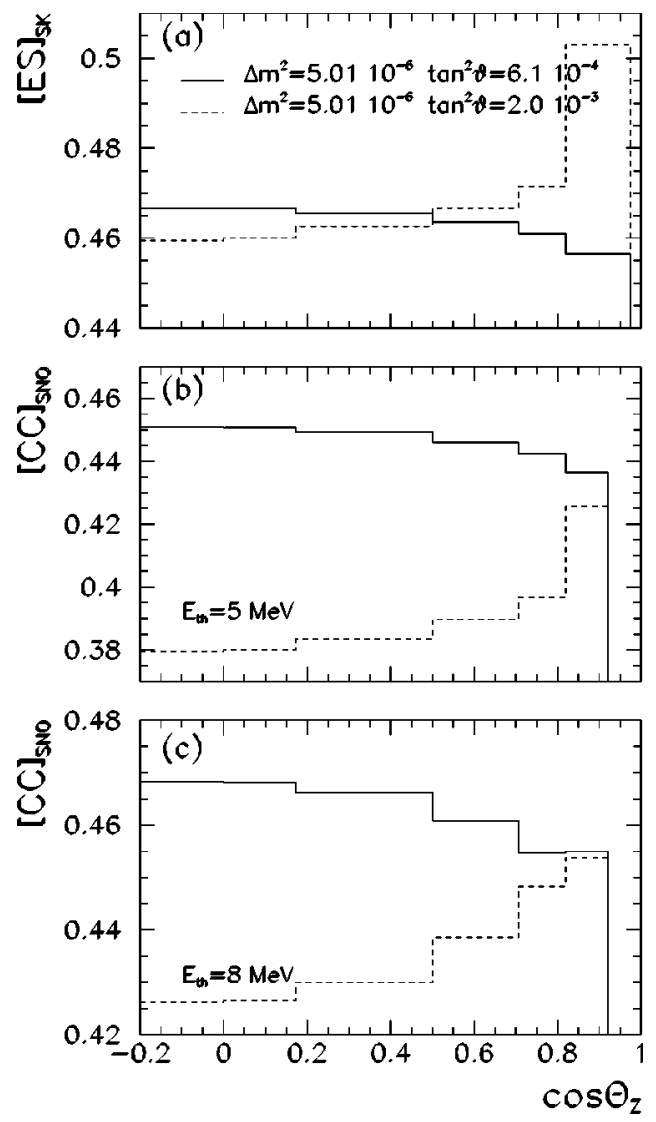

FIG. 9. Same as Fig. 8 for the SMA solution.

section and the regeneration factor increase with $E$. As a result, the main contribution to the integrated regeneration factor, $\left\langle f_{\text {reg }}\left(\theta_{Z}\right)\right\rangle$, comes from a rather narrow interval of energies: $E=9-13 \mathrm{MeV}$, so that $\Delta E / E \sim 1 / 3$. For the central value of the energy $E=11 \mathrm{MeV}$ and oscillation parameters in the best fit point (see Fig. 3) the oscillation length is about $10^{3} \mathrm{~km}$. This distance corresponds to $\cos \theta_{Z} \sim 0.07$. That is, for neutrinos propagating at $\cos \theta_{Z} \sim 0.07$ the oscillation phase will be $\phi \approx 2 \pi$ and $f_{\text {reg }}$ will be at a minimum. In general, we get from Eq. (31)

$$
\phi=14 \pi \cos \theta_{Z}\left(\frac{\Delta m^{2}}{3 \times 10^{-5} \mathrm{eV}^{2}}\right)\left(\frac{10 \mathrm{MeV}}{E}\right) .
$$

Therefore, the phase difference $\Delta \phi$ for neutrinos which cross the Earth in the direction $\cos \theta_{Z}$ and differ in energy by $\Delta E$ is

$$
\Delta \phi \approx \phi \frac{\Delta E}{E} \approx 2 \pi \frac{\cos \theta_{Z}}{0.07} \frac{\Delta E}{E}
$$

If $\Delta \phi \sim 2 \pi$ one would expect strong averaging effect. From this condition and using Eq. (38) with $\Delta E / E=1 / 3$ we find that strong averaging should appear at $\cos \theta_{Z} \approx 0.2$ in agreement with the results of the numerical calculations, as seen in Figs. 3(b) and 3(c). For large $\cos \theta_{Z}$, the waves are again synchronized, so that a constructive interference of the waves leads to the restoration of the oscillatory behavior. 

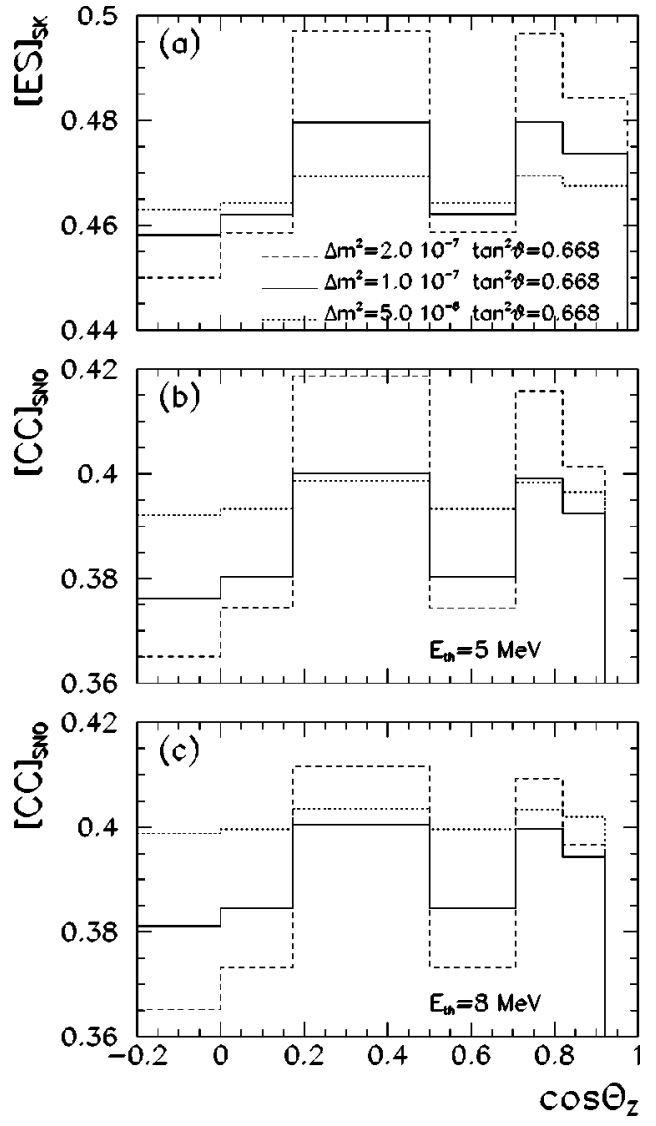

FIG. 10. Same as Fig. 8 for the LOW solution.

From Eq. (38) we also see that the periods of constructive and destructive interference become shorter with the increase of $\Delta m^{2}$ (see Fig. 4). Also with the increase of the energy threshold the relevant energy interval $\Delta E$ becomes narrower and, correspondingly, the period of synchronization becomes longer (in $\cos \theta_{Z}$ scale) as can be seen in Figs. 3(c) and 4(c).

\section{B. SMA}

In the SMA region we have $\cos 2 \theta_{S} \approx-1$. Furthermore, the adiabaticity is broken inside the Sun: $P_{c} \neq 0$, so that

$$
P_{e e}=P_{D}+\left(1-2 P_{c}\right) f_{\text {reg }} .
$$

Notice that since in the SMA region $\cos 2 \theta \sim 1$, one has $P_{D}$ $\approx P_{c}$. SK data imply that $P_{c} \approx P_{D}=0.35-0.65$, therefore in the SMA region the regeneration effect is substantially suppressed by the adiabaticity factor: $\left|1-2 P_{c}\right|<0.3$. For $P_{c}$ $>1 / 2$ the regeneration effect becomes negative.

In Fig. 5(a) we show the zenith angle dependence of the regeneration factor for different values of neutrino energy and for oscillation parameters corresponding to the best fit point in the SMA region. The behavior of the curves can be understood taking into account that in the SMA region

$$
\eta=0.3-3
$$

so the SMA solution region covers the resonance range for the Earth matter densities. For the best fit point we have

$$
l_{\nu} \approx l_{0} \approx(4-8) \times 10^{3} \mathrm{~km}
$$

For the mantle region $\left(\cos \theta_{Z}<0.837\right)$ we find the following.

(1) Far from the resonance, $\eta>1$, the oscillation length,

$$
l_{m} \approx \frac{l_{\nu} \eta}{|1-\eta|}
$$

is smaller than the Earth diameter. However the amplitude of oscillations is strongly suppressed [see the line which corresponds to the $E=5 \mathrm{MeV}$ in Fig. 5(a)].

(2) In the resonance, $\eta=1$, the amplitude of oscillations is strongly enhanced, but the oscillation length

$$
l_{m} \approx \frac{l_{\nu}}{\sin ^{2} 2 \theta} \approx 10^{5} \mathrm{~km}
$$

is much larger than the Earth diameter. As a result, the oscillation effect is small (the line for $E=12 \mathrm{MeV}$ in Fig. 5). In the small phase limit the regeneration factor equals

$$
f_{\text {reg }} \approx \sin ^{2} 2 \theta \frac{\pi^{2} D^{2} \cos ^{2} \theta_{Z}}{l_{\nu} l_{0}} .
$$

It increases quadratically with $\cos \theta_{Z}$.

(3) The line for $E=15 \mathrm{MeV}$ represents an intermediate case when the phase of oscillations is about $\pi$ for $\cos \theta_{Z}$ $\approx 0.8$. In this case the regeneration factor equals

$$
f_{\text {reg }} \approx 4 \sin ^{2} 2 \theta \frac{D^{2} \cos ^{2} \theta_{Z}}{l_{\nu} l_{0}} \approx 4 \sin ^{2} 2 \theta \frac{D^{2} \cos ^{2} \theta_{Z}}{l_{0}^{2}},
$$

where in the second equality we have taken into account that $\eta \sim 1$. Since $l_{0} \approx 0.4 D$ for large $\cos \theta_{Z}$ trajectories in the mantle, the mixing enhancement factor, $4\left(D / l_{0}\right)^{2}$, can be as large as 25 , so that $f_{\text {reg }} \approx 0.04$ for $\sin ^{2} 2 \theta=2.4 \times 10^{-3}$ and $\cos \theta_{Z}=0.8$.

Clearly, the adiabaticity is strongly broken near the resonance: $l_{m} \gg l_{\rho}$. But far from the resonance: $l_{m} \sim l_{\rho} \sim D$, the violation of the adiabaticity is moderate, so one can describe its effect as oscillations in a narrow layer with some effective density.

For $\cos \theta_{Z}>0.837$ neutrinos cross the core of the Earth. For the core densities the resonance energies are in the range $E=3-5 \mathrm{MeV}\left(\Delta m^{2} \sim 5 \times 10^{-6} \mathrm{eV}^{2}\right)$. For energies between $E_{R}$ (core) and $E_{R}$ (mantle), the parametric enhancement of oscillations takes place leading to the appearance of the parametric peak in the $\cos \theta_{Z}$ distribution of $f_{\text {reg }}$. For SMA the realization of the parametric resonance corresponds to a mixing angle in mantle smaller than maximal mixing, $2 \theta_{\text {mantle }}$ $<\pi / 2$, while in the core, $2 \theta_{\text {core }}>\pi / 2$. At the peak the relation between the oscillation phases in the mantle $\phi_{\text {mantle }}$ and in the core $\phi_{\text {core }}$ corresponds to the general condition for the parametric resonance $[17,20,21]$

$$
X_{3} \equiv s_{m} c_{c} \cos 2 \theta_{\text {mantle }}+s_{c} c_{m} \cos 2 \theta_{\text {core }} \approx 0 \text {, }
$$


where $s_{m} \equiv \sin \left(\phi_{\text {mantle }} / 2\right), c_{c} \equiv \cos \left(\phi_{\text {mantle }} / 2\right)$. For the best fit point $\tan ^{2} \theta=6.1 \times 10^{-4}, \Delta m^{2}=5 \times 10^{-6} \mathrm{eV}^{2}$, and $E=10$ $\mathrm{MeV}$ we find that the maximum of the parametric peak occurs at $\cos \theta_{Z}=0.863$. The phases in the core and in the mantle are $\phi_{\text {mantle }}=0.49 \pi, \phi_{\text {core }}=0.49 \pi$, and the effective mixing angles are $2 \theta_{\text {mantle }}=0.188$ and $\pi-2 \theta_{\text {core }}=0.113$. Then we get $X_{3} \sim 0.045 \ll 1$.

As illustration, in Fig. 2(b) we show the dependence of the regeneration factor on the distance for the parameters which correspond to the maxima of the parametric peaks.

In Figs. 5(a) and 6(a) we show the dependence of the regeneration factor on the mixing angle. Both the oscillation length and the resonance condition depend on $\sin ^{2} 2 \theta$ weakly, so that with a good precision $f_{\text {reg }} \propto \sin ^{2} 2 \theta$. Notice that this proportionality holds also for core crossing trajectories.

In Figs. 5 and 6 [panels (b) and (c)] we show the zenith angle dependence of the event rates at SK and SNO. The integration over energy basically reproduces the dependence of $f_{\text {reg }}$ on $\cos \theta_{Z}$ for $E \sim 10 \mathrm{MeV}$ since this energy gives the dominant contribution. The $[\mathrm{CC}]$ event rate at SNO can be writen as

$$
[\mathrm{CC}] \approx\left\langle P_{D}\right\rangle_{C C}+\left(1-2 \bar{P}_{c}\right)\left\langle f_{\mathrm{reg}}\left(\theta_{Z}\right)\right\rangle_{C C},
$$

where $\bar{P}_{c}$ is an effective jump probability for the contributing energies. (Notice that $P_{c}$ changes slower with $E$ than $f_{\text {reg }}$ does.)

For $\tan ^{2} \theta \sim 6 \times 10^{-4}$ (see Fig. 5) the adiabaticity breaking is strong, $\bar{P}_{c}>1 / 2$ and the negative adiabatic factor leads to the suppression of the rate due to Earth-matter effect. On the other hand, for $\tan ^{2} \theta \sim 2.0 \times 10^{-3}$ (see Fig. 6), the adiabaticity breaking is weaker, $\bar{P}_{c}<1 / 2$, and the Earth-matter effect is positive. As follows from the figures, the adiabatic factor suppresses substantially the regeneration effect:

$$
\left|1-2 \bar{P}_{c}\right| \frac{\left\langle f_{\text {reg }}\left(\theta_{Z}\right)\right\rangle_{C C}}{f_{\text {reg }}} \lesssim 0.2-0.3 .
$$

The $[\mathrm{CC}]$ rate depends weakly on the energy threshold in the mantle region but it strongly decreases with $E_{t h}$ in the core region. The parametric peak is wider (in energy scale) than the resonance peak in the mantle and therefore the integration over the energy leads to a stronger decrease of the regeneration effect in the mantle region than in the core region. Also the parametric peak is situated at the low energy part of the spectrum: $(5-10 \mathrm{MeV})$. As a consequence, the core peak decreases substantially with the increase of the threshold from 5 to $8 \mathrm{MeV}$.

\section{LOW}

In the LOW solution region one has $\cos 2 \theta_{S} \approx-1$, and moreover, to good approximation we can take $P_{c} \approx 0$, so that

$$
P_{e e} \approx \sin ^{2} \theta+f_{\text {reg }} \text {. }
$$

The zenith angle dependence of the regeneration factor for oscillation parameters in the best fit point and different values of the neutrino energy are shown in Fig. 7(a). The interpretation of the results is rather straightforward. In the LOW region we have $\eta<0.1$. In particular, in the best fit point at $E=10 \mathrm{MeV}: \eta<0.03$. Therefore the oscillations proceed in the matter dominated regime. Thus, $l_{\nu} \gg l_{0}$ and the oscillation length is determined mainly by the refraction length. Notice that in the limit $\eta \ll 1$ we get from Eq. (18)

$$
l_{m} \approx l_{0}(1+2 \eta \cos 2 \theta)
$$

Moreover, in the LOW region the mixing parameter is small: $\cos 2 \theta<0.5$ at $99 \%$ C.L. (in the best fit point: $\cos 2 \theta \approx 0.2$ ). So, the correction to $l_{0}$ is further suppressed, for instance, $2 \eta \cos 2 \theta \sim 0.012$ for $E=10 \mathrm{MeV}$. Therefore for a given trajectory, the oscillation phase is practically independent of the neutrino energy and mixing angle [see different curves in Fig. 7(a)].

The phase of the oscillatory behavior of $f_{\text {reg }}$ with $\cos \theta_{Z}$ can be written as

$$
\phi=\sqrt{2} G_{F} \int_{0}^{D \cos \theta_{Z}} n_{e}\left(x, \cos \theta_{Z}\right) d x=\frac{2 \pi D \cos \theta_{Z}}{\bar{l}_{0}\left(\cos \theta_{Z}\right)},
$$

where $\bar{l}_{0}\left(\cos \theta_{Z}\right)$ is the average refraction length along the trajectory determined by $\cos \theta_{Z}$ and $n_{e}=\rho Y_{e} / m_{N}$ is the electron number density. With increase of $\cos \theta_{Z}$, the average density increases so, $\bar{l}_{0}$ decreases and the period of oscillations becomes shorter. Thus the oscillation phase is determined by $\cos \theta_{Z}$. The first maximum of $f_{\text {reg }}(\phi=\pi)$ is achieved at $\cos \theta_{Z}=0.35$, the minimum $(\phi=2 \pi)$ lies at $\cos \theta_{Z}=0.59$ and second maximum occurs at $\cos \theta_{Z}=0.8$ (see also [7]).

The oscillation length is comparable with the scale of density variations $l_{m} \approx l_{0} \sim 8 \times 10^{3} \mathrm{~km}$, where $l_{\rho} \sim D / 2$, so that the adiabaticity is broken moderately. Since $l_{m}$ is comparable with the size of the layer, the effect can be considered as oscillation in the layer of matter with some effective constant density.

The amplitude of oscillations,

$$
A_{f} \approx \eta \sin ^{2} 2 \theta \approx \frac{\sqrt{2} \Delta m^{2} \sin ^{2} 2 \theta}{G_{F} E \bar{n}\left(\cos \theta_{Z}\right)}
$$

is proportional to $\Delta m^{2}$ and inversely proportional to $E$. It is also inversely proportional to the average density for a given trajectory $\bar{n}$ which increases with $\cos \theta_{Z}$. Correspondingly the second peak is lower. For $E=10 \mathrm{MeV}$ and oscillation parameters at the best fit point we get $\eta=0.2$ (for the surface density), and consequently according to Eq. (52) the height of the first peak is expected to be $f_{\text {reg }}=0.042$ which is slightly larger than the numerical result $f_{\text {reg }}=0.035$ shown in Fig. 7(a). The difference of the results is due to the adiabaticity breaking.

Combining Eqs. (51) and (52) we get an approximate expression for the regeneration factor in the mantle region: 


$$
f_{\mathrm{reg}} \approx \frac{\sqrt{2} \sin ^{2} 2 \theta}{G_{F} \bar{n}\left(\cos \theta_{Z}\right)} \frac{\Delta m^{2}}{E} \sin ^{2}\left[\frac{G_{F}}{\sqrt{2}} \int_{0}^{D \cos \theta_{Z}} n_{e}\left(x, \cos \theta_{Z}\right) d x\right] .
$$

Notice also that $f_{\text {reg }}$ increases with $\sin ^{2} 2 \theta$.

In the core region $f_{\text {reg }}$ is enhanced due to the parametric effect. Indeed, we find that for $\cos \theta_{Z}=0.92$, which corresponds to the position of the third maximum, the phases of oscillations equal

$$
\phi_{\text {mantle }}=0.98 \pi, \quad \phi_{\text {core }}=2.98 \pi .
$$

That is, they are very close to $\pi$ and $3 \pi$

$$
\phi_{\text {mantle }} \approx \pi, \quad \phi_{\text {core }} \approx 3 \pi .
$$

At this condition in the constant density approximation the height of the peak would reach the value (see Appendix)

$$
\begin{aligned}
f_{\text {reg }} & \sim \sin ^{2}\left(\theta-4 \theta_{\text {mantle }}+2 \theta_{\text {core }}\right)-\sin ^{2} \theta \\
& \approx \sin 2 \theta \sin ^{2}\left(4 \theta_{\text {mantle }}-2 \theta_{\text {core }}\right),
\end{aligned}
$$

where $\theta_{\text {mantle }}$ and $\theta_{\text {core }}$ are the mixing angles in the mantle and in the core correspondingly, and the last equality is valid for $\theta_{\text {mantle }} \sim \theta_{\text {core }} \sim \pi / 2$. The peak in Fig. 7 (a) is slightly lower than what Eq. (56) gives because of the density change. Notice that this realization of the parametric enhancement corresponds to both mixing angles above the resonance, (see [30] and Appendix). The real time evolution of the neutrino state (dependence of the regeneration factor on distance) for the parameters corresponding to the peak is shown in Fig. 2(c).

In Fig. 7(b) and Fig. 7(c) we show the zenith angle dependence of [ES] event rates and [CC] event rates at SK and SNO respectively. The $[\mathrm{CC}]$ rate has the same approximate expression as in Eq. (35) for the LMA solution. Since $f_{\text {reg }}$ $\propto 1 / E$ and the phase of oscillations depends very weakly on $E$, we get $\left\langle f_{\text {reg }}\right\rangle_{C C} \approx f_{\text {reg }}(\bar{E})$, where $\bar{E} \sim(10-11) \mathrm{MeV}$ is the effective energy of the spectrum. Therefore the [CC] distribution reproduces all the features of the zenith angle dependence of $f_{\text {reg }}$. Furthermore, the regeneration term weakly decreases with the increase of the threshold energy from 5 to $8 \mathrm{MeV}$, and it increases with mixing as $\left\langle f_{\text {reg }}\right\rangle_{C C} \propto \sin ^{2} 2 \theta$ according to Eq. (53).

For the [ES] distribution at SK, the results are similar to those for [CC] with the only additional feature of a $25 \%$ damping effect due to the contribution from $\nu_{\mu} / \nu_{\tau}$ scattering via NC [see approximate formula in Eq. (36)].

\section{IDENTIFICATION OF THE SOLUTION: CORRELATIONS}

\section{A. Binned rates}

According to Figs. 3-7 the LMA, SMA, and LOW solutions present qualitatively different zenith angle dependence of the event rates (either [ES] or [CC]).

(1) For the LMA solution one expects a significant rate already at $\cos \theta_{Z} \sim 0.03$. The first peak is at $\cos \theta_{Z}$ $=0.02-0.03$. The rate has an oscillatory behavior at small, $\cos \theta_{Z}<0.2$, and large $\cos \theta_{Z}>0.6-0.8$, zenith angles. The border of the oscillatory region at high $\theta_{Z}$ depends on the value of the mass difference. For instance, for $\Delta m^{2} \sim 2.5$ $\times 10^{-5} \mathrm{eV}^{2}$ the second oscillatory region starts at $\cos \theta_{Z}$ $\sim 0.8$ while for $\Delta m^{2} \sim 4.5 \times 10^{-5} \mathrm{eV}^{2}$ it expands down to $\cos \theta_{Z} \sim 0.6$. The period of oscillations is small, $\Delta\left(\cos \theta_{Z}\right)$ $<0.05-0.07$, and in consequence, it will be difficult to detect the oscillatory behavior due to the relatively low statistics. Although we find that for small values of $\Delta m^{2}$ the amplitude of oscillations can reach $15 \%$ of the average rate. When averaging over wider $\cos \theta_{Z}$ bins, the binned rate depends rather weakly on $\cos \theta_{Z}$ and no significant change of the rate is expected in the core region.

(2) For the SMA solution the rate changes slowly and monotonously in the mantle region and significant parametric effects can appear in the core region. For the large $\tan ^{2} \theta$ the rate increases with $\cos \theta_{Z}$ and the parametric enhancement leads to the appearance of the parametric peak which gives the main contribution to the integral regeneration effect $[7,8,10,4,5]$. For small $\tan ^{2} \theta$ the rate decreases with $\cos \theta_{Z}$ and in the core one has a dip in the distribution of events.

(3) The LOW solution predicts the existence of three peaks in the zenith angle distribution of events. There are two oscillation peaks in the mantle range: a wide peak with the maximum at $\cos \theta_{Z}=0.35$ and a narrow peak at $\cos \theta_{Z}$ $\sim 0.8$. The third peak (in the core region) is due to the parametric enhancement of oscillations. The maximum of this peak is at $\cos \theta_{Z} \sim 0.92$.

These features are rather generic. The qualitative behavior of the distribution is the same for all points within a given solution, although quantitatively the sizes of the different structures appearing in the distributions change with the oscillation parameters. Therefore, in principle a detailed study of the zenith angle distributions will not only give the identification of the solution of the problem but also the determination of the neutrino oscillation parameters.

Due to the relatively small present statistics (effects themselves are small) it is unavoidable to bin the distribution of events. The binning should be chosen in such a way to avoid as much as possible the averaging or washing out of the structures.

We propose the following binning. We will enumerate the night bins as $\mathrm{N} 1 \ldots \mathrm{N} 5$ and denote by $[\mathrm{N}]_{i},(i=1 \ldots 5)$ the average rate in a given bin Ni normalized to the no oscillation prediction. We will call it the reduced rate. We will denote by $[\mathrm{N}]$ and $[\mathrm{D}]$ the averaged reduced rates during the night and during the day correspondingly.

Since in the mantle range both the SMA and LMA solutions predict a rather flat distribution with weak dependence on $\cos \theta_{Z}$, we suggest the binning which emphasizes the features of the distribution for the LOW solution.

Bin N1: $\cos \theta_{Z}=0-0.173\left(90^{\circ}>\theta_{Z}>80^{\circ}\right)$. In this bin one expects very small regeneration effect both for the SMA and LOW solutions. Significant effect should be observed in the case of the LMA solution.

Bin N2: $\cos \theta_{Z}=0.173-0.5\left(80^{\circ}>\theta_{Z}>60^{\circ}\right)$. This bin is selected in such a way that it covers the main part of the wide peak of the LOW distribution. 
Bin N3: $\cos \theta_{Z}=0.5-0.707\left(60^{\circ}>\theta_{Z}>45^{\circ}\right)$. This bin covers the dip of the LOW distribution: $[\mathrm{N}]_{3}(\mathrm{LOW}) \sim[\mathrm{D}]$.

Bin N4: $\cos \theta_{Z}=0.707-0.83\left(45^{\circ}>\theta_{Z}>35^{\circ}\right)$ is the last mantle bin. This bin corresponds to the second peak of the LOW solution.

Bin N5: $\cos \theta_{Z}>0.83\left(\theta_{Z}<35^{\circ}\right)$, the core bin. This bin is restricted by $\cos \theta_{Z}=0.83-0.975$ for SK and $\cos \theta_{Z}$ $=0.83-0.92$ for SNO. For LMA the rate is comparable with the rates in the previous bins. For the LOW solution one expects to see a rate slightly smaller than the one in the fourth bin. Here the regeneration effect due to the parametric enhancement of oscillations takes place. For SMA the effect depends strongly on the oscillation parameters: for large $\tan ^{2} \theta$ one should see the highest rate. With decrease of the mixing angle the rate decreases and becomes smaller than the day rate.

In Figs. 8, 9, and 10 we show the binned event rates for [ES] events at SK and [CC] events at SNO for oscillation parameters in the LMA, SMA, and LOW solutions respectively. The distributions are rather similar for [ES] events at SK and $[\mathrm{CC}]$ events at SNO. Although the enhancement of the Earth-matter effect in the core bin for the SMA solution is larger in SK, since this bin covers a larger interval of $\cos \theta_{Z}$ at the SK latitude.

The qualitative behavior of the $[\mathrm{CC}]$ rates does not change with the increase of the threshold energy from $5 \mathrm{MeV}$ to $8 \mathrm{MeV}$ for SNO. The absolute value of the effect (reduced rate), however, increases for the LMA solution and it decreases for the LOW and SMA solutions.

For the LMA solution the regeneration effect decreases with the increase of $\Delta m^{2}$ in all the bins and it changes weakly with the mixing. For the LOW solution the situation is opposite: the rates $[\mathrm{N}]_{2},[\mathrm{~N}]_{4}$, and $[\mathrm{N}]_{5}$ increase with $\Delta m^{2}$. This behavior originates from the dependence of $f_{\text {reg }}$ on $\Delta m^{2}$ [see Eqs. (53) and (56)]. Also for SMA the rate in the core bin increases with $\tan ^{2} \theta$.

The comparison of the event rates in the different bins allows to discriminate among the solutions of the solar neutrino problem. In particular, for LMA we have

$$
[\mathrm{N}]_{1}<[\mathrm{N}]_{2} \leqslant[\mathrm{~N}]_{3} \leqslant[\mathrm{~N}]_{4} \leqslant[\mathrm{~N}]_{5} .
$$

For LOW distribution:

$$
[\mathrm{N}]_{2} \geqslant[\mathrm{~N}]_{4}>[\mathrm{N}]_{1} \sim[\mathrm{N}]_{3}>[\mathrm{D}] .
$$

For SMA distribution:

$$
\begin{aligned}
\left|[\mathrm{N}]_{1}-[\mathrm{D}]\right| & <\left|[\mathrm{N}]_{2}-[\mathrm{D}]\right| \\
& \leqslant\left|[\mathrm{N}]_{3}-[\mathrm{D}]\right| \leqslant\left|[\mathrm{N}]_{4}-[\mathrm{D}]\right| \\
& \leqslant\left|[\mathrm{N}]_{5}-[\mathrm{D}]\right| .
\end{aligned}
$$

Finally, for the sake of completeness we show in Fig. 11 the time exposure for the different bins (average during the year) for the SK and SNO detectors. The histograms are normalized in such a way that the integral over night time gives 0.5 . The time exposure distribution determines the statistics in each bin. As follows from the figure the highest statistics is expected in N2 followed by N3. Moreover, at SNO the time

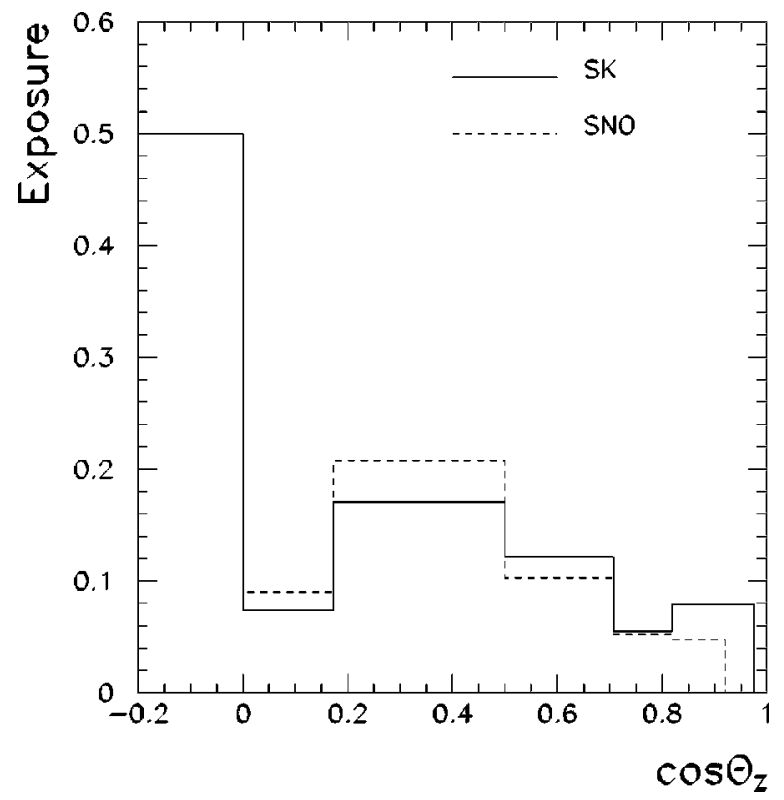

FIG. 11. The exposure time for the different $\cos \theta_{Z}$ bins for SNO and SK detectors during the year.

exposure N2 will be about twice longer than N3, while for SK the difference in statistics is smaller. For SNO the lowest statistics is expected in N5, whereas for SK it corresponds to N4.

\section{B. Correlations}

At present, the expected regeneration effects are restricted by the Super-Kamiokande result on the day-night asymmetry:

$$
A_{\mathrm{DN}}^{\mathrm{SK}}=2 \frac{N-D}{N+D} \sim 0.034 \pm 0.026
$$

The asymmetry gives the integrated (over $\cos \theta_{Z}$ ) Earthmatter effect. With present statistics the integral effect is at $1.3 \sigma$ level which means that in spite of large number of events it will be difficult to measure the zenith angle distribution with a high precision. Higher asymmetry (due to absence of damping) is expected for SNO [32]:

$$
A_{\mathrm{ND}}^{\mathrm{SNO}}<0.10-0.15 \text {. }
$$

To enhance the identification power of the analysis one can study the correlations between the signals in different zenith angle bins and the integral effect which can be represented by the day-night asymmetry. As an illustration of such correlations we first show in Fig. 12 contours of constant asymmetry $A_{\mathrm{DN}}$ and of ratios of the reduced rates $[\mathrm{N}]_{2} /[\mathrm{N}]_{3}$ and $[\mathrm{N}]_{5} /[\mathrm{N}]_{2}$ in the $\Delta m^{2}-\tan ^{2} \theta$ plane for SNO with threshold $5 \mathrm{MeV}$.

For the LMA solution the $\cos \theta_{Z}$ distribution is rather flat which means that for all bins

$$
\frac{[\mathrm{N}]_{\mathrm{i}}}{[\mathrm{N}]}-1 \propto A_{\mathrm{DN}}
$$




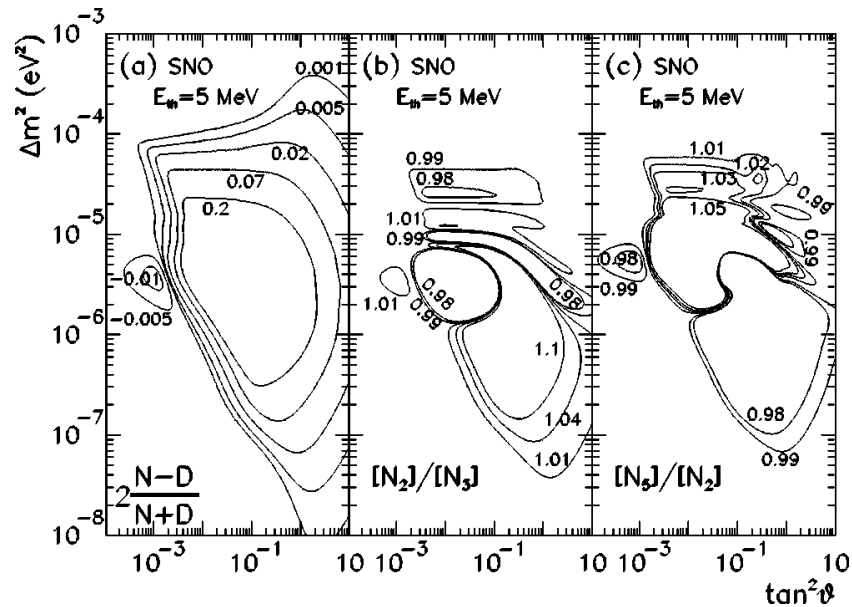

FIG. 12. The contours of equal day-night asymmetry, $A_{\mathrm{DN}}$ (a), the ratios of rates $N_{5} / N_{2}$ (b), and $N_{2} / N_{3}$ (c) at SNO (threshold 5 $\mathrm{MeV})$ in the $\Delta m^{2}-\tan ^{2} \theta$ plane.

For the LOW solution the heights of all three peaks increase with integral effect:

$$
\frac{[\mathrm{N}]_{2}}{[\mathrm{~N}]}-1 \propto A_{\mathrm{DN}}
$$

and similar proportionality exists for $[\mathrm{N}]_{4}$ and $[\mathrm{N}]_{5}$. In the third bin, however, the regeneration effect is practically zero and the rate is at the level of the day rate.

For the SMA solution, the rate monotonously increases (or decreases depending on $\tan \theta$ ) with $\cos \theta_{Z}$, so that for all the bins the proportionality (62) holds approximately.

\section{Ratio $[\mathrm{N}]_{5} /[\mathrm{N}]_{2}$ versus $A_{\mathrm{DN}}$}

Comparing the signals in N2 and N5 bins we get

$$
\frac{[\mathrm{N}]_{5}}{[\mathrm{~N}]_{2}} \sim 1 \quad(\mathrm{LMA}), \quad \frac{[\mathrm{N}]_{5}}{[\mathrm{~N}]_{2}}>1 \quad(\mathrm{LOW}) .
$$

In both these cases the day-night asymmetry is positive. On the other hand for SMA we have $[\mathrm{N}]_{5} /[\mathrm{N}]_{2}>1$ for the part of the allowed region with small mixing, for $A_{\mathrm{DN}}<0$, and $[\mathrm{N}]_{5} /[\mathrm{N}]_{2}<1$ for the part of the region with large mixing, where $A_{\mathrm{DN}}>0$ (see Fig. 12).

In Figs. 13 and 14 we show the results of mapping the $99 \%$ C.L. solution regions onto the plane of the $[\mathrm{N}]_{5} /[\mathrm{N}]_{2}$ $-A_{\mathrm{DN}}$ observables for the SNO and SK experiments. From the figures we see that there is a strong correlation between the $[\mathrm{N}]_{5} /[\mathrm{N}]_{2}$ ratio and the asymmetry for all three solutions.

For SNO observables above $5 \mathrm{MeV}$ (Fig. 13) the correlation can be parametrized as

$$
\frac{[\mathrm{N}]_{5}}{[\mathrm{~N}]_{2}} \approx \begin{cases}1 & \text { LMA } \\ 1-0.45 A_{\mathrm{DN}} & \text { LOW } \\ 1+3.6 A_{\mathrm{DN}} & \text { SMA }\end{cases}
$$

A negative asymmetry would testify for the SMA solution but the maximal asymmetry which is expected for the SMA

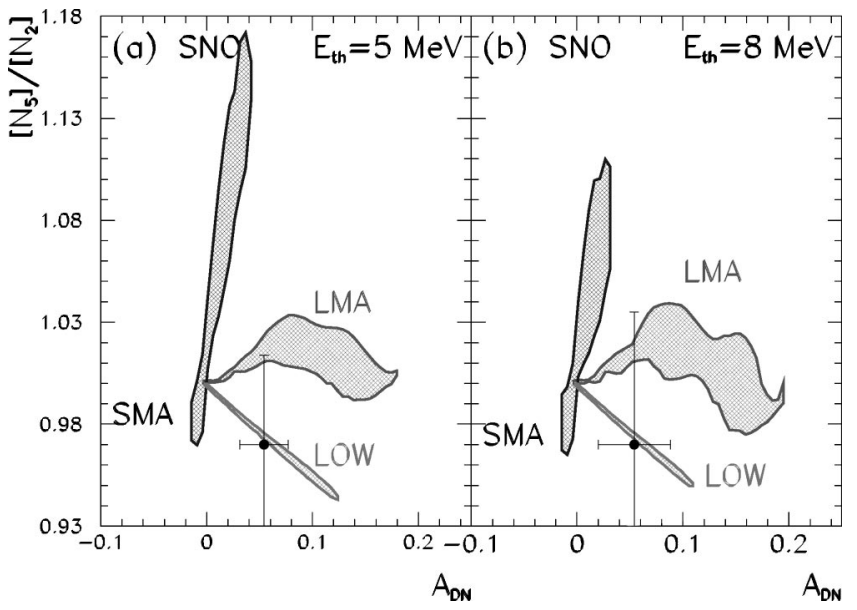

FIG. 13. The allowed values for the day-night asymmetry, $A_{\mathrm{DN}}$, and the ratio of rates in the N5 and N2 bins, $N_{5} / N_{2}$, at SNO above $5 \mathrm{MeV}$ (a) and $8 \mathrm{MeV}$ (b) in the different allowed regions (at 99\% C.L.) of the solar neutrino problem. We also show the expected statistical sensitivity after 3 years of operation (see text for details).

on the basis of the present data cannot be larger than 0.05 . Thus, for $A_{\mathrm{DN}}(\mathrm{SNO})>0.05$ (above $5 \mathrm{MeV}$ ) one will choose between LMA and LOW. A ratio $[\mathrm{N}]_{5} /[\mathrm{N}]_{2}>1$ will testify for LMA, whereas $[\mathrm{N}]_{5} /[\mathrm{N}]_{2}<1$ will be an evidence of the LOW solution. For $A_{\mathrm{DN}}=0$, the equality $[\mathrm{N}]_{5} /[\mathrm{N}]_{2}=1$ is fulfilled for all the solutions. In the range $A_{\mathrm{DN}}(\mathrm{SNO})<0.05$ all three solutions are possible. With increase of the asymmetry the difference of ratios for different solutions increases.

For the SNO measurements with $E_{t h}=8 \mathrm{MeV}$, the correlations are described approximately by Eq. (65), but the LMA region has a more irregular shape since at $E_{t h}=8 \mathrm{MeV}$ the averaging is weaker. The maximal allowed deviations of $[\mathrm{N}]_{5} /[\mathrm{N}]_{2}$ from 1 are $\sim 11 \%(\mathrm{SMA}), \sim 4 \% \quad(\mathrm{LMA})$ and $\sim-5 \%$ (LOW).

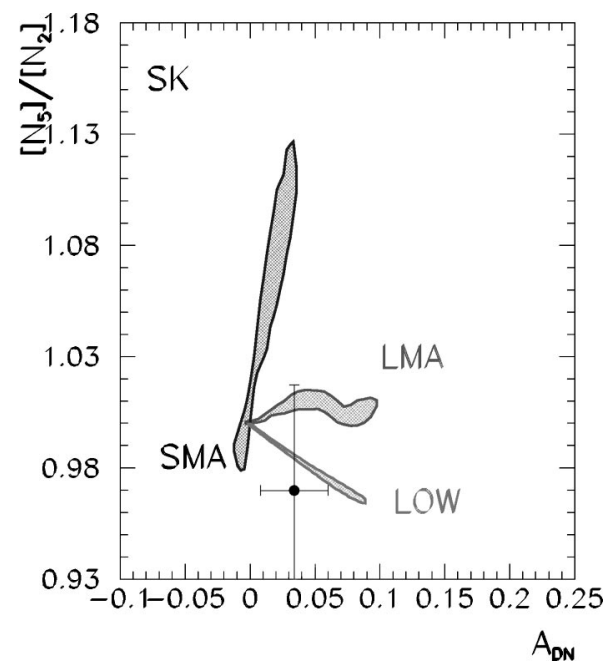

FIG. 14. The allowed values for the day-night asymmetry, $A_{\mathrm{DN}}$, and the ratio of rates in the $\mathrm{N} 5$ and $\mathrm{N} 2$ bins, $N_{5} / N_{2}$, at SK in the different allowed regions (at $99 \%$ C.L.) of the solar neutrino problem. The present experimental values and $1 \sigma$ errors are also displayed (see text for details). 


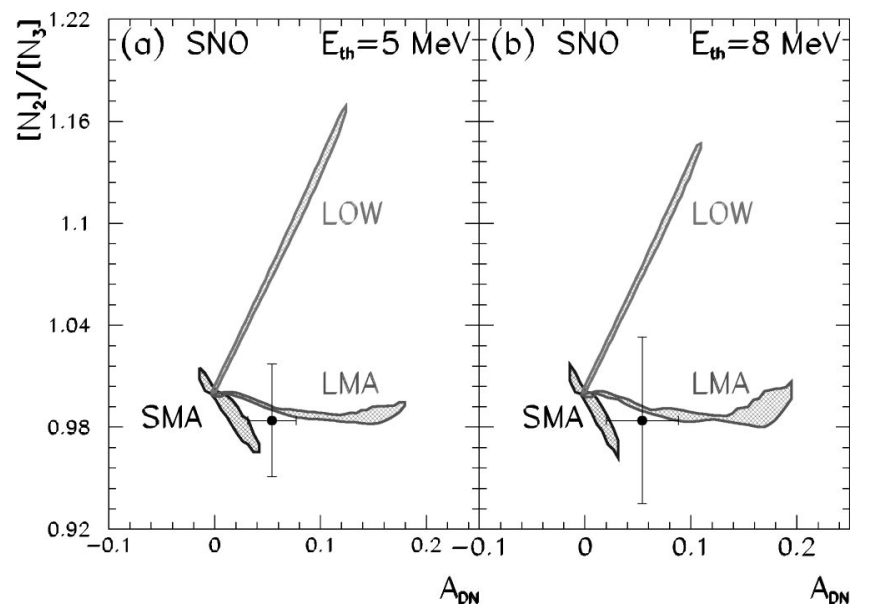

FIG. 15. The allowed values for the day-night asymmetry, $A_{\mathrm{DN}}$, and the ratio of rates in the $\mathrm{N} 2$ and $\mathrm{N} 3$ bins, $N_{2} / N_{3}$, at SNO above $5 \mathrm{MeV}$ (a) and $8 \mathrm{MeV}$ (b) in the different allowed regions (at 99\% C.L.) of the solar neutrino problem. We also show the expected statistical sensitivity after 3 years of operation (see text for details).

Comparing with the results at lower energy threshold we find that the allowed SMA region in $[\mathrm{N}]_{5} /[\mathrm{N}]_{2}-A_{\mathrm{DN}}$ plane shrinks and $A_{\mathrm{DN}}(\mathrm{SNO})$ can reach only 0.04 , while $[\mathrm{N}]_{5} /[\mathrm{N}]_{2}$ can still be as large as 1.11 . This happens because the region of parametric enhancement is less covered with the higher threshold. For the LOW solution the allowed region increases (since the regeneration effect decreases with energy). $A_{\mathrm{DN}}(\mathrm{SNO})$ can reach $11 \%$ and $\left(N_{5} / N_{2}-1\right) \sim 0.05$.

For the ES events at SK (Fig. 14) one gets a similar picture, although the regeneration effects are damped by the $\nu_{\mu}$ and $\nu_{\tau}$ contribution. The expected maximal asymmetry is $A_{\mathrm{DN}}(S K) \sim 3 \%$ for SMA and $A_{\mathrm{DN}}(\mathrm{SK}) \sim 10 \%$ for LMA and LOW. If $A_{\mathrm{DN}}(\mathrm{SK}) \sim 5 \%$, the $[\mathrm{N}]_{5} /[\mathrm{N}]_{2}$ ratio for LMA is larger than the ratio for LOW by $(0.03-0.05)$.

To have an idea about present sensitivity in searches of correlations, we also show in Fig. 14 the SK results and with $1 \sigma$ errors for the day-night asymmetry and the $[\mathrm{N}]_{5} /[\mathrm{N}]_{2}$ ratio. One must bare in mind, however, that the plotted result on $[\mathrm{N}]_{5} /[\mathrm{N}]_{2}$ does not correspond to the binning we are proposing. In Fig. 13 we show the corresponding attainable SNO sensitivity for three years of operation (corresponding to $13200 \mathrm{CC}$ events for $5 \mathrm{MeV}$ threshold and $6000 \mathrm{CC}$ events for $8 \mathrm{MeV}$ threshold). For definiteness we have plotted the point at a value $A_{\mathrm{DN}}(\mathrm{SNO})=1.6 A_{\mathrm{DN}}(\mathrm{SK})$ which is the expected relation for the best fit point in LMA as discussed in Ref. [32].

\section{Ratio $[\mathrm{N}]_{2} /[\mathrm{N}]_{3}$ versus $A_{\mathrm{DN}}$}

According to Eqs. (57)-(59) (see also Fig. 12), we have

$$
\begin{gathered}
{[\mathrm{N}]_{2} \approx[\mathrm{N}]_{3} \quad(\mathrm{LMA}), \quad[\mathrm{N}]_{2}>[\mathrm{N}]_{3} \quad(\mathrm{LOW}),} \\
\left|[\mathrm{N}]_{2}-[\mathrm{D}]\right|<\left|[\mathrm{N}]_{3}-[\mathrm{D}]\right| \quad(\mathrm{SMA}) .
\end{gathered}
$$

So that the rates in the high statistics bins N2 and N3 can provide an important criteria to distinguish the solutions.

In Fig. 15 we show the mapping the 99\% C.L. allowed

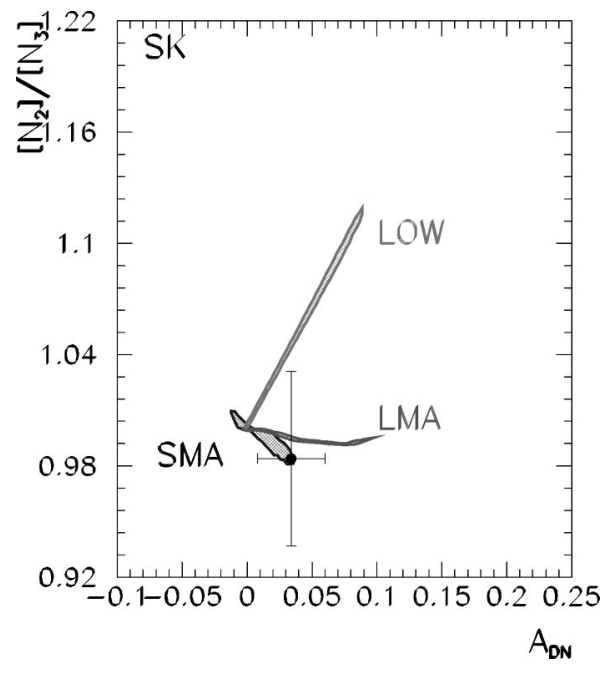

FIG. 16. The allowed values for the day-night asymmetry, $A_{\mathrm{DN}}$, and the ratio of rates in the $\mathrm{N} 3$ and $\mathrm{N} 2$ bins, $N_{3} / N_{2}$, at SK in the different allowed regions (at $99 \%$ C.L.) of the solar neutrino problem. The present experimental values and $1 \sigma$ errors are also displayed (see text for details).

regions of oscillation parameters onto the $[\mathrm{N}]_{2} /[\mathrm{N}]_{3}-A_{\mathrm{DN}}$ plane of the SNO observables for two different energy thresholds. From the figure one can see that there is a clear correlation between the ratio and the asymmetry for the SMA and LOW solutions:

$$
\frac{[\mathrm{N}]_{2}}{[\mathrm{~N}]_{3}} \approx \begin{cases}1 & \text { LMA, } \\ 1+1.3 A_{\mathrm{DN}} & \text { LOW, } \\ 1-1.1 A_{\mathrm{DN}} & \text { SMA. }\end{cases}
$$

Also, we see that the regions only weakly depend on the energy threshold. In the case of the LOW solution the $[\mathrm{N}]_{2} /[\mathrm{N}]_{3}$ ratio can reach 1.17 for $E_{t h}=5 \mathrm{MeV}$ and 1.15 for $E_{t h}=8 \mathrm{MeV}$. For SMA the maximal allowed deviation of the $[\mathrm{N}]_{2} /[\mathrm{N}]_{3}$ ratio from 1 is about $4 \%$. We get qualitatively similar results for SK (Fig. 16), where the regeneration effect is damped by $\nu_{\mu}$ and $\nu_{\tau}$ contributions, so that the allowed regions for all three solutions are smaller than for SNO. We also plot in Figs. 16 and 15 the present results from SK and the estimated accuracy at SNO as discussed at the end of the previous section.

From these results it is clear that, with the estimated statistics at SK and SNO, it will be difficult to get strong discrimination among the solutions. At $3 \sigma$ level all the solutions seem to be allowed. However, future higher statistics experiments like UNO [33] can give a more significant result.

One may also attempt to elaborate a test criteria for some of the solutions without a large loss of statistics. For instance, for the LOW solution one expects a significant deviation from one for the ratio

$$
\frac{[\mathrm{N}]_{2}+[\mathrm{N}]_{4}+[\mathrm{N}]_{5}}{[\mathrm{~N}]_{1}+[\mathrm{N}]_{3}}>1
$$

while for LMA this ratio should be close to one. 


\section{CONCLUSIONS}

In this work we have studied in detail the zenith angle dependence of the regeneration factor and the distributions of the solar neutrino events expected in the SuperKamiokande and SNO detectors.

We have identified the generic features of the distributions for each solution.

(1) The LMA $\cos \theta_{Z}$ distribution is characterized by a fast oscillatory behavior with rather slowly changing averaged regeneration rate. The amplitude of oscillations is enhanced in the core region. The behavior of the event rates at SNO and SK has an interesting "synchronization effect"' so that strong oscillations of the rates with the change of $\cos \theta_{Z}$ are only expected at small and at large values of $\cos \theta_{Z}$. In particular 2-3 oscillations with significant amplitude are expected at small $\cos \theta_{Z}$.

To detect such a behavior one needs to use small size binning $\Delta\left(\cos \theta_{Z}\right) \sim 0.05$ which (in view of relatively low statistics) may only be possible with new megaton water Cherenkov detectors. If the LMA solution is identified, a study of the oscillatory behavior can be used to measure the oscillation parameters (in particular $\Delta m^{2}$ ) and the Earth density profile.

(2) The SMA solution predicts a smooth zenith angle dependence in the mantle region with small regeneration effect while the parametric effects can show up in the core region. For the larger mixing part of the allowed region the regeneration effect is positive with a parametric peak in the core region; for the smaller mixings the effect is negative and in the core region one should see the parametric dip.

(3) In the case of the LOW solution there are two oscillation peaks in the mantle region with maxima at $\cos \theta_{Z}$ $=0.35$ (oscillation phase $\phi=\pi$ ) and at $\cos \theta_{Z}=0.78$ (the phase $3 \pi$ ). In the core region the third peak appears due to parametric enhancement of oscillations. In the maximum the conditions for the parametric resonance are approximately satisfied. This realization of the parametric resonance corresponds to the case of neutrino energy above the resonance energy in the mantle (which differs from the realization for the SMA) and to the phase relations $\phi_{\text {mantle }} \approx \pi$ and $\phi_{\text {core }}$ $\approx 3 \pi$. We find that the position of the peaks is almost independent of the neutrino energy, whereas the height of the peaks as well as the integral effect are inversely proportional to the energy.

The shape of the distributions and their dependence on the oscillation parameters as well as energy thresholds are well described by oscillations in a medium which consists of one or three layers with slowly changing density. Such an oscillation picture gives the correct functional dependence of the rates on the neutrino parameters.

We also show that precise measurements of the zenith angle distribution will allow to study a number of various matter effects such as oscillation in matter with constant and slowly changing density, adiabatic conversion, parametric enhancement of oscillations, etc.

We have suggested a new binning in the zenith angle distribution which is chosen to emphasize the distinctive features of the distributions for the different solutions. The iden- tification power of the analysis can be further enhanced by studying correlations between the event rates in the different bins and the integral effect which can be represented by the day-night asymmetry. In particular we have shown that the study of the correlation between the ratio $[\mathrm{N}]_{2} /[\mathrm{N}]_{3}$ and the day-night asymmetry will help to identify the LOW solution while the correlation between $[\mathrm{N}]_{5} /[\mathrm{N}]_{2}$ and $A_{\mathrm{D} / \mathrm{N}}$ is more suitable for identification of the SMA solution. The distributions expected at SNO and at SK have similar properties. But at SNO the structures in the distributions and the asymmetry are enhanced due to the absence of the damping effect.

\section{ACKNOWLEDGMENTS}

This work was supported by the Spanish DGICYT under grants PB98-0693 and PB97-1261, by the Generalitat Valenciana under grant GV99-3-1-01 and by the TMR network grant ERBFMRXCT960090 of the European Union.

\section{APPENDIX: GRAPHIC REPRESENTATION OF EVOLUTION OF THE NEUTRINO STATE}

We describe here a graphic representation of the evolution of the neutrino state which allows us to understand the properties of the zenith angle distributions for the different solutions. The graphic representation [19] is based on the analogy of the neutrino evolution with the behavior of spin of the electron in the magnetic field. The neutrino state is described by a vector of length $1 / 2$ with components

$$
\vec{\nu}=\left(\operatorname{Re} \psi_{e}^{\dagger} \psi_{\mu}, \operatorname{Im} \psi_{e}^{\dagger} \psi_{\mu}, \psi_{e}^{\dagger} \psi_{e}-1 / 2\right),
$$

where $\psi_{i},(i=e, \mu)$ are the neutrino wave functions of the electron and muon neutrinos. (The components of this vector are elements of the density matrix.)

Introducing the vector

$$
\vec{B} \equiv \frac{2 \pi}{l_{m}}\left(\sin 2 \theta_{m}, 0, \cos 2 \theta_{m}\right)
$$

( $\theta_{m}$ is the mixing angle and $l_{m}$ is the oscillation length in the medium) which would correspond to the magnetic field, one can get from the evolution equation for $\psi_{i}$ the equation for $\vec{\nu}$ :

$$
\frac{d \vec{\nu}}{d t}=(\vec{\nu} \times \vec{B})
$$

According to Eq. (A1) the projection of $\vec{\nu}$ on the axis $z$, $\nu_{z}$, gives the probability to find $\nu_{e}$ in the state $\vec{\nu}$ :

$$
P \equiv \psi_{e}^{\dagger} \psi_{e}=\nu_{z}+\frac{1}{2}=\cos ^{2} \frac{\theta_{e}}{2}
$$

Here $\nu_{z} \equiv 0.5 \cos \theta_{e}$, and $\theta_{e}$ is the angle between $\vec{\nu}$ and the axis $z$. The $z$ axis can be called the flavor axis as it coincides with vectors $\vec{\nu}_{e}=-\vec{\nu}_{\mu}$. 

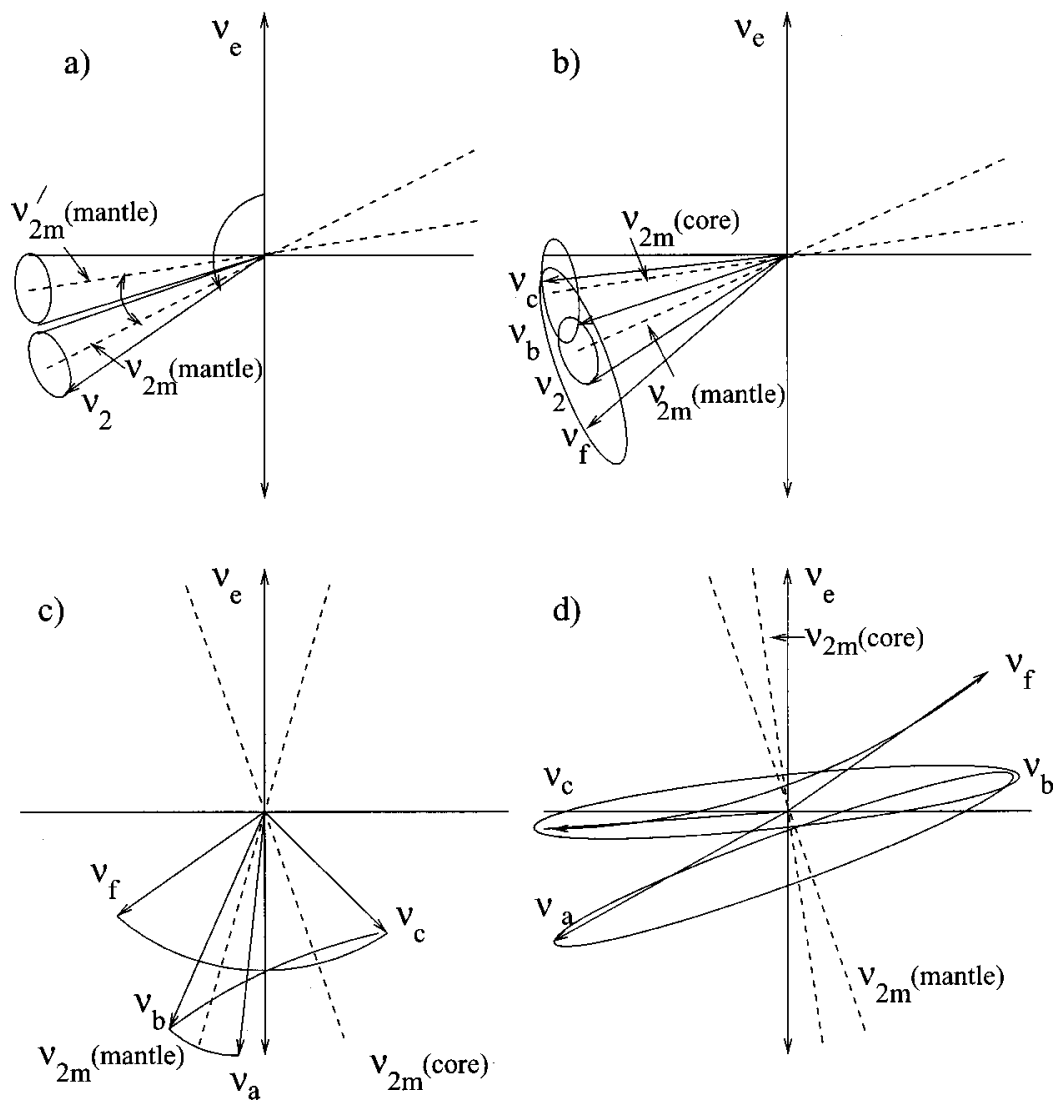

FIG. 17. Graphic representation of the evolution of the neutrino state inside the Earth for different solutions of the solar neutrino problem. (a) LMA solution: propagation along trajectory which crosses the mantle only; (b) LMA solution: evolution for core crossing trajectory; (c) SMA solution: evolution for core crossing trajectory and $\cos \theta_{Z}$ which corresponds to the maximum of the parametric peak; (d) the same as in (c) for LOW solution.
In a medium with constant density, $\theta_{m}=$ const, the evolution corresponds to the precession of the vector $\vec{\nu}$ around $\vec{B}$ : $\vec{\nu}$ moves according to the increase of the oscillation phase, $\phi$, on the surface of the cone with axis $\vec{B}$. The vector $\vec{B}$ coincides with the vector of the eigenstates in matter $\vec{\nu}_{1 \mathrm{~m}}$ $=-\vec{\nu}_{2 m}$. We will denote by $\vec{\nu}_{2 m}$ (mantle) and $\vec{\nu}_{2 m}$ (core) the axis in the mantle and in the core. Notice that when the matter density is below the resonant one $(\eta>1)$ the corresponding axis lies in the first-third quadrants with decreasing projection on the $z$ axis as the density increases [see Figs. 17(a) and 17(b)]. Once the resonant density is crossed, the axis moves to the second-fourth quadrants [see Figs. 17(c) and $17(\mathrm{~d})]$.

In Figs. 17(a) and Figs. 17(b) we show the evolution of the neutrino state in the case of the LMA solution for trajectories crossing the mantle only (a) or the mantle and the core (b). Inside the Sun the neutrino vector (produced as $\vec{\nu}=\nu_{e}$ $\approx \vec{\nu}_{2 m}$ ) follows the vector $\vec{\nu}_{2 m}$ due to adiabaticity and appears at the surface of the Sun and then at the surface of the Earth as $\vec{\nu}=\vec{\nu}_{2}$. Due to the adiabatic density change in the mantle the axis $\vec{\nu}_{2 m}$ moves accordingly, but at the detector it (as well as the cone of precession) will return to the same position as in the moment of arrival of the neutrino at the Earth. The cones with axis $\vec{\nu}_{2 m}$ and $\vec{\nu}_{2 m}^{\prime}$ describe oscillations at the surface of the Earth and in the central part of the trajectory correspondingly.

At the surface of the Earth the density jumps suddenly and therefore the axis vector $\vec{\nu}_{2 m}$ suddenly changes its position from $\vec{\nu}_{2}$ to $\vec{\nu}_{2 m}$ (mantle). In the mantle the neutrino vector will precess around $\vec{\nu}_{2 m}$ (mantle). Clearly, since $\vec{\nu}_{2 m}$ (mantle) is closer than $\vec{\nu}_{2}$ to $\nu_{e}$, the rotation will lead to an increase of the $z$ projection and therefore to increase the $P_{e e}$ probability. This illustrates the fact that the regeneration is always positive in the mantle zone (see also [31]). The quasiadiabatic movements of $\vec{\nu}_{2 m}$ (mantle) due to density variations in the mantle do not change this conclusion.

Let us now consider the evolution for the core crossing trajectory. The neutrino vector starts at $\vec{\nu}_{a}=\nu_{2}$ and it will first precess around $\vec{\nu}_{2 m}$ (mantle). The phase of oscillations is rather large $\sim(10-20) \pi$ and it depends sensitively on the neutrino energy and the zenith angle. So, depending on the energy the neutrino state can arrive at the core in any position on the cone. In Fig. 17(b), as an example, we have selected the state $\vec{\nu}_{b}$. In the core the vector $\vec{\nu}$ will precess around $\vec{\nu}_{2 m}$ (core) starting from the position $\vec{\nu}_{b}$. The phase is large and the state will enter the mantle again in some position $\vec{\nu}_{c}$ which depends on $E$ and $\theta_{Z}$. In the second mantle layer the state will precess around $\vec{\nu}_{2 m}$ (mantle) starting from $\vec{\nu}_{c}$ state. Let us denote by $\vec{\nu}_{f}$ the final neutrino state at the detector. As it is clear from the figure, in most of configurations determined by the positions of $\vec{\nu}_{c}$ and $\vec{\nu}_{b}$ (and therefore $E$ and $\theta_{z}$ ) the final state will have the projection $\vec{\nu}_{z}$ larger that the projection of $\vec{\nu}_{a}=\nu_{2}$ (which corresponds to day signal). In other words: $\cos 2 \theta_{e}\left(\vec{\nu}_{f}\right)<\cos 2 \theta$. In some cases, however, one can get $\nu_{z}$ (final) $<\nu_{2 z}$ which corresponds to negative regeneration.

It is easy to see that maximal regeneration effect would correspond to zero regeneration effect in the mantle: $\phi_{\text {mantle }}=2 \pi k$ and to maximal effect in the core: $\phi_{\text {core }}=\pi$ $+2 \pi k^{\prime}$ ( $k, k^{\prime}$ are integer numbers). 
In Fig. 17(c) we show the evolution of the neutrino state in the case of SMA solution. Now the neutrino arrives at the Earth as an incoherent superposition of $\nu_{1}$ and $\nu_{2}$. This split of the state originates from the adiabaticity violation inside the Sun. The total survival probability $P_{e e}$ is then determined by independent oscillations of $\nu_{1}$ and $\nu_{2}$ inside the Earth. The result can be represented in terms on $\nu_{2} \rightarrow \nu_{e}$ probability and the adiabaticity factor (12). The latter can have both negative and positive signs depending on the oscillation parameters, and it is this factor which determines the sign of the regeneration effect.

In Fig. 17(c) we show the evolution of the $\nu_{2}$ state inside the Earth. In the mantle the vector $\vec{\nu}$ precesses around $\vec{\nu}_{2 m}$ (mantle) starting from $\vec{\nu}_{a}=\nu_{2}$. In the core region, the parametric enhancement of oscillations occurs for certain values of $\cos \theta_{Z}$. We show the graphic representation of evolution for this case. Neutrino arrives at the core in the state $\vec{\nu}_{b}$, then $\vec{\nu}$ precesses around $\vec{\nu}_{2 m}$ (core) starting from $\vec{\nu}_{b}$ and reaches the border of core and mantle in the state $\vec{\nu}_{c}$ (which corresponds to the phase $\phi_{\text {core }} \sim 0.75 \pi$ ). In the second mantle layer $\vec{\nu}$ rotates around $\vec{\nu}_{2 m}$ (mantle) starting from $\vec{\nu}_{c}$ and arrive at the detector in the state $\vec{\nu}_{f}$. Clearly, the regeneration turns out to be enhanced.

In the case of the LOW solution [Fig. 17(d)], $\vec{\nu}$ arrives at the Earth as $\vec{\nu}_{a} \approx \nu_{2}$ similarly to the LMA case but in contrast to LMA, now the mixing is matter suppressed so that the axis of the eigenstates is close to the flavor axis $(z)$. In the mantle $\vec{\nu}$ precesses around $\vec{\nu}_{2 m}$ (mantle) starting from position $\vec{\nu}_{a}$. The peaks in the zenith angle distribution in the mantle region correspond to the final position of the neutrino $\vec{\nu}_{b}$. In the core region the parametric enhancement of oscillations occurs. The maximum of the parametric peak corresponds to the following picture. Vector $\vec{\nu}$ precesses in the mantle and reaches the state $\vec{\nu}_{b}$ at the border of the core $\left(\phi_{\text {mantle }} \approx \pi\right)$. In the core $\vec{\nu}$ rotates around $\vec{\nu}_{2 m}$ (core) starting from $\vec{\nu}_{b}$. The neutrino vector make 1.5 turns $\left(\phi_{\text {core }}=3 \pi\right)$ and leave the core in the position $\vec{\nu}_{c}$. In the second mantle layer $\vec{\nu}$ rotates again around $\vec{\nu}_{2 m}$ (mantle) with initial position $\vec{\nu}_{c}$. At the detector one will detect the state which corresponds to $\vec{\nu}_{f}$.
[1] S. P. Mikheyev and A. Yu. Smirnov, in '86 Massive Neutrinos in Astrophysics and in Particle Physics, Proceedings of the Sixth Moriond Workshop, edited by O. Fackler and J. Trân Thanh Vân (Editions Frontières, Gif-sur-Yvette, 1986), p. 355 .

[2] J. Bouchez et al., Z. Phys. C 32, 499 (1986); M. Cribier et al., Phys. Lett. B 182, 89 (1986); E. D. Carlson, Phys. Rev. D 34, 1454 (1986).

[3] A. J. Baltz and J. Weneser, Phys. Rev. D 35, 528 (1987); A. Dar et al., ibid. 35, 3607 (1987); S. P. Mikheyev and A. Yu. Smirnov, Usp. Fiz. Nauk. 153, 30 (1987) [Sov. Phys. Usp. 30, 759 (1987)]; M. L. Cherry and K. Lande, Phys. Rev. D 36, 3571 (1987); S. Hiroi, H. Sakuma, T. Yanagida, and M. Yoshimura, Phys. Lett. B 198, 403 (1987); Prog. Theor. Phys. 78, 1428 (1987); A. J. Baltz an d J. Weneser, Phys. Rev. D 37, 3364 (1988); M. Spiro and D. Vignaud, Phys. Lett. B 242, 297 (1990).

[4] J. N. Bahcall and P.I. Krastev, Phys. Rev. C 56, 2839 (1997).

[5] Q. Y. Liu, M. Maris, and S. T. Petcov, Phys. Rev. D 56, 5991 (1997); M. Maris and S. T. Petcov, ibid. 56, 7444 (1997); M. Narayan, G. Rajasekaran, and R. Sinha, Mod. Phys. Lett. A 13, 1915 (1998); A. de Gouvea, A. Friedland, and H. Murayama, hep-ph/9910286; G. L. Fogli, E. Lisi, and D. Montanino, Phys. Rev. D 61, 073009 (2000); G. L. Fogli, E. Lisi, D. Montanino, and A. Palazzo, ibid. 62, 113003 (2000).

[6] M. Maris and S. T. Petcov, Phys. Rev. D 62, 093006 (2000).

[7] A. J. Baltz and J. Weneser, Phys. Rev. D 50, 5971 (1994); 51, 3960 (1994).

[8] N. Hata and P. Langacker, Phys. Rev. D 48, 2937 (1993); 50, 632 (1994).

[9] E. Lisi and D. Montanino, Phys. Rev. D 56, 1792 (1997).

[10] J. M. Gelb, Wai-kwok Kwong, and S. P. Rosen, Phys. Rev. Lett. 78, 2296 (1997).
[11] J. N. Bahcall, P. I. Krastev, and A. Yu. Smirnov, Phys. Rev. D 60, 093001 (1999).

[12] P. C. de Holanda, C. Pena-Garay, M. C. Gonzalez-Garcia, and J. W. F. Valle, Phys. Rev. D 60, 093010 (1999).

[13] A. H. Guth, L. Randall, and M. Serna, J. High Energy Phys. 08, 018 (1999).

[14] M. C. Gonzalez-Garcia, C. Peña-Garay, Y. Nir, and A. Yu. Smirnov, Phys. Rev. D 63, 013007 (2001).

[15] J. M. Losecco, Phys. Rev. D 47, 2032 (1993).

[16] S. T. Petcov, Phys. Lett. B 434, 321 (1998).

[17] E. Kh. Akhmedov, Nucl. Phys. B538, 25 (1999).

[18] V. K. Ermilova, V. A. Tsarev, and V. A. Chechin, Kr. Soob. Fiz. 5, 26 (1986); E. Kh. Akhmedov, Yad. Fiz. 47, 475 (1988) [Sov. J. Nucl. Phys. 47, 301 (1988)].

[19] P. I. Krastev and A. Yu. Smirnov, Phys. Lett. B 226, 341 (1989).

[20] M. V. Chizhov and S. T. Petcov, Phys. Rev. Lett. 83, 1096 (1999).

[21] E. K. Akhmedov and A. Yu. Smirnov, Phys. Rev. Lett. 85, 3978 (2000).

[22] S. T. Petcov, Phys. Lett. B 200, 373 (1988).

[23] P. I. Krastev and S. T. Petcov, Phys. Lett. B 207, 64 (1988).

[24] E. K. Akhmedov, hep-ph/0011136.

[25] A. M. Dziewonski and D. L. Anderson, Phys. Earth Planet. Inter. 25, 297 (1981).

[26] J. N. Bahcall, M. Kamionkowsky, and A. Sirlin, Phys. Rev. D 51, 6146 (1995).

[27] S. Nakamura, T. Sato, V. Gudkov, and K. Kubodera, Phys. Rev. C . 63, 034617 (2001); see also, http://nuc003.psc.sc.edu/ $\sim$ kubodera

[28] http://www.sns.ias.edu/ jnb/SNdata/Export/BP2000; J. N. Bahcall, S. Basu, and M. H. Pinsonneault, Astrophys. J. 529, 1084 (2000).

[29] M. C. Gonzalez-Garcia and C. Peña-Garay, Phys. Rev. D 63, 073013 (2001), and references therein. 
[30] Q. Y. Liu and A. Yu. Smirnov, Nucl. Phys. B524, 505 (1998); Q. Y. Liu, S. P. Mikheyev, and A. Yu. Smirnov, Phys. Lett. B 440, 319 (1998).

[31] C. W. Chiang and L. Wolfenstein, Phys. Rev. D 63, 057303 (2001).
[32] J. N. Bahcall, P. I. Krastev, and A. Yu. Smirnov, Phys. Rev. D 62, 093004 (2000); 63, 053012 (2001).

[33] C. K. Jung, hep-ex/0005046; see also http:// superk.physics.sunysb.edu/ uno/ 